\title{
q-DEFORMATION OF WITT-BURNSIDE RINGS
}

\author{
YOUNG-TAK OH
}

\begin{abstract}
In this paper, we construct a $q$-deformation of the Witt-Burnside ring of a profinite group over a commutative ring, where $q$ ranges over the set of integers. When $q=1$, it coincides with the Witt-Burnside ring introduced by A. Dress and C. Siebeneicher (Adv. Math. 70 (1988), 87-132). To achieve our goal we first show that there exists a $q$-deformation of the necklace ring of a profinite group over a commutative ring. As in the classical case, i.e., the case $q=1, q$-deformed Witt-Burnside rings and necklace rings always come equipped with inductions and restrictions. We also study their properties. As a byproduct, we prove a conjecture due to Lenart (J. Algebra. 199 (1998), 703-732). Finally, we classify $\mathbb{W}_{G}^{q}$ up to strict natural isomorphism in case where $G$ is an abelian profinite group.
\end{abstract}

\section{INTRODUCTION}

The universal ring of Witt vectors was introduced by Witt and Lang around 1965. In [10], they discovered that there exists a unique covariant functor $\mathbb{W}$ on the category of commutative rings with identity characterized by the following properties:

(1) As a set, it is $A^{\mathbb{N}}$.

(2) For any ring homomorphism $f: A \rightarrow B$, the map $\mathbb{W}(f): \mathbf{a} \mapsto\left(f\left(a_{n}\right)\right)_{n \geq 1}$ is a ring homomorphism for $\mathbf{a}=\left(a_{n}\right)_{n \geq 1}$.

(3) The maps $w_{m}: \mathbb{W}(A) \rightarrow A$ defined by

$$
\mathbf{a} \mapsto \sum_{d \mid m} d a_{d}^{\frac{m}{d}} \text { for } \mathbf{a}=\left(a_{n}\right)_{n \geq 1}
$$

are ring homomorphisms $([2,8,10])$

Here, $\mathbb{W}(A)$ is called the universal ring of Witt vectors over $A$.

The theory of Witt vectors has been developed with deep connection with necklace rings, which are algebraic objects arising from the combinatorics of necklaces. To be more precise, in [12], Metropolis and Rota introduced a covariant functor $\mathrm{Nr}$ on the category of commutative rings with identity. In this case, $\operatorname{Nr}(A)$ is called the necklace ring over $A$. They then showed that

$$
\mathbb{W}(\mathbb{Z}) \cong N r(\mathbb{Z}) \text {. }
$$

To do this they studied the combinatorics of necklaces extensively and described Witt vectors in terms of strings, which are infinite matrices satisfying suitable conditions.

The author gratefully acknowledges support from the following grants: KOSEF Grant \# R012003-000-10012-0; KRF Grant \# 2006-331-C00011.

MSC : 11F03,11F22,17B70.

Keywords: Witt-vectors, Necklace ring, Witt-Burnside ring, Burnside-Grothendieck ring 
On the other hand, in [7], Dress and Siebeneicher observed that $N r(\mathbb{Z})$ is naturally isomorphic to the Burnside-Grothendieck ring $\hat{\Omega}(C)$ of isomorphism classes of almost finite cyclic sets. Here, $C$ denotes the multiplicative infinite cyclic group. Motivated by this fact, they found that given any profinite group, say $G$, there exists a unique covariant functor $\mathbb{W}_{G}$ on the category of commutative rings with identity, satisfying

$$
\text { i) } \mathbb{W}_{G}(\mathbb{Z}) \cong \hat{\Omega}(G) \text { and } \text { ii) } \mathbb{W}_{\hat{C}}=\mathbb{W} \text {. }
$$

Here, $\hat{\Omega}(G)$ denotes the Burnside-Grothendieck ring of $G$ of isomorphism classes of almost finite $G$-spaces, and $\hat{C}$ the profinite completion of $C$. In this case, $\mathbb{W}_{G}(A)$ is called the Witt-Burnside ring of $G$ over A.

Recently, in $[13,16]$, it was shown that given any profinite group $G$, there exists a unique covariant functor $N r_{G}$ from the category of special $\lambda$-rings to the category of commutative rings with identity, satisfying

$$
\mathbb{W}_{G}(A) \cong N r_{G}(A)
$$

where $A$ is any special $\lambda$-ring. Obviously, $N r_{\hat{C}}$ is not equivalent to $N r$ since domains are different. This led us to introduce $\widehat{N r}_{G}$, which is a unique covariant functor on the category of commutative rings with identity, satisfying

$$
\text { i) } \widehat{N r}_{G}(\mathbb{Z}) \cong \hat{\Omega}(G) \text { and ii) } \widehat{N r}_{\hat{C}}=N r \text {. }
$$

In view of ii), $\widehat{N r}_{G}$ may be regarded as a group-theoretic generalization of $N r$. Furthermore, viewed as a functor from the category of binomial rings to the category of commutative rings with identity, $N r_{G}$ and $\widehat{N r}_{G}$ turn out to be same $([15,16])$.

The theory of Witt vectors arises in the context of formal group laws, too ([8]). It is well-known that for every one-dimensional formal group law $F(X, Y)$ over a torsion-free ring $B$, there exists a unique covariant functor $\mathbb{W}^{F}$ from the category of $B$-algebras to the category of abelian groups. Related to this functor, it is quite surprising that if $F(X, Y)$ is given by $X+Y-q X Y, q \in \mathbb{Z}$, then the value of $\mathbb{W}^{F}$, usually denoted by $\mathbb{W}^{q}$, at each object has the structure of a commutative ring. This phenomenon was first observed by Lenart ([11]) for commutative torsion-free rings with identity. Later, in [15], it was shown that his observation makes sense for arbitrary commutative rings. Consequently, one can derive a covariant functor $\mathbb{W}^{q}$ from the category of commutative rings to itself in this case. In particular, if $q=1$, then it coincides with $\mathbb{W}$. In this sense, we may regard $\mathbb{W}^{q}$ as a $q$-deformation of $\mathbb{W}$.

Since $\mathbb{W}=\mathbb{W}_{\hat{C}}$, it would be quite natural to expect that the same phenomenon also happens to the functor $\mathbb{W}_{G}$ for arbitrary profinite groups. Recall that the key ingredient in proving the existence of $\mathbb{W}_{G}$ is that the polynomials determining the ring structure of $\mathbb{W}_{G}(\mathbb{Z})$ have integer coefficients. These polynomials were utilized to endow $\mathbb{W}_{G}(A)$ with the ring structure for arbitrary commutative rings with identity. In contrast, the polynomials arising from the construction of $\mathbb{W}_{G}^{q}$, the $q$-deformation of $\mathbb{W}_{G}$, are in $\mathbb{Q}[q]$. The most significant and difficult step in our construction will be to show that these polynomials take integer values for integer arguments. To do this we first introduce $N r_{G}^{q}$, the $q$-deformation of $N r_{G}$, and then define $q$-inductions and $q$-restrictions. Exploiting $q$-inductions and $q$-restrictions, we construct an isomorphism, called $q$-Teichmüller map. With this preparation, we finally show that Dress and Siebeneicher's proof can be applied to our case. 
This paper is organized as follows. In Section 2, we introduce the functors $\mathbb{W}_{G}$, $N r_{G}$ and $\widehat{N r}_{G}$. In Section 3, we introduce orbit-sum polynomials associated with a profinite group, which play the same role as necklace polynomials in the theory of Witt vectors. In Section $4, N r_{G}^{q}$ and $\widehat{N r}_{G}^{q}$ will be defined as $q$ ranges over the set of integers, and in Section 5, natural transformations called $q$-induction and $q$-restriction will be defined. Main results will appear in Section $6 . \mathbb{W}_{G}^{q}$ will be defined as $q$ ranges over the set of integers, and many structural properties will be investigated. Section 7 is devoted to proving lemmas and theorems which are stated without proof. In particular, we prove a conjecture due to Lenart([11]) concerning $q$-restriction and $q$-necklace polynomials. In the final section, we show that $\mathbb{W}_{G}^{q}$ is classified up to strict natural isomorphism by $\mathrm{D}^{\mathrm{pr}}(q) \cap \mathrm{D}^{\mathrm{pr}}(G)$ as $q$ ranges over the set of integers. Here, $\mathrm{D}^{\mathrm{pr}}(q)$ denotes the set of prime divisors of $q$, and $\mathrm{D}^{\mathrm{pr}}(G)$ the set of prime divisors of each of

$$
\{(G: U) \mid U \text { is an open subgroup of } G\} .
$$

\section{The Witt- Burnside Ring And the necklace Ring of A Profinite GROUP}

In this section, we review prerequisites on the Witt- Burnside ring and the necklace ring of a profinite group. For more information see $[13,16]$. Unless otherwise stated, the rings we consider will be commutative, but not necessarily unital.

Let $G$ be an arbitrary profinite group. For any $G$-space $X$ and any subgroup $U$ of $G$ define $\varphi_{U}(X)$ to be the cardinality of the set $X^{U}$ of $U$-invariant elements of $X$ and let $G / U$ denote the $G$-space of left cosets of $U$ in $G$. For two subgroups $U, V$ of $G$, we say that $U$ is subconjugate to $V$ if $U$ is a subgroup of some conjugates of $V$. This is a partial order on the set of the conjugacy classes of open subgroups of $G$, and will be denoted by $[V] \preceq[U]$. Fix an enumeration of this poset satisfying the condition:

$$
\text { If }[V] \preceq[U] \text {, then }[V] \text { precedes }[U] \text {. }
$$

By abuse of notation we denote this poset by $\mathcal{O}(G)$. For example, if $G$ is abelian*, then $\mathcal{O}(G)$ is just the set of open subgroups of $G$ subject to

$$
V \preceq U \Longleftrightarrow U \subseteq V .
$$

Given a commutative ring $A$ and a profinite group $G$, we define the ghost ring of $G$ over $A$, denoted by $\operatorname{Gh}(G, A)$, to be the commutative ring

$$
A^{\mathcal{O}(G)}\left(:=\prod_{i \in \mathcal{O}(G)} A\right)
$$

whose addition and multiplication are defined componentwise.

Definition 2.1. ([6]) Given a profinite group $G, \mathbb{W}_{G}$ is a unique covariant functor from the category of commutative rings with identity to itself characterized as follows.

(1) As a set

$$
\mathbb{W}_{G}(A)=A^{\mathcal{O}(G)}
$$

\footnotetext{
* In case where $G$ is abelian, we omit the bracket notation.
} 
(2) For every ring homomorphism $f: A \rightarrow B$ and every $\mathbf{x} \in \mathbb{W}_{G}(A)$ one has

$$
\mathbb{W}_{G}(f)(\mathbf{x})=f \circ \mathbf{x} \text {. }
$$

(3) The map,

$$
\Phi: \mathbb{W}_{G}(A) \rightarrow \mathrm{Gh}(G, A), \quad \mathbf{x} \mapsto\left(\sum_{[G] \preceq[V] \preceq[U]} \varphi_{U}(G / V) \cdot \mathbf{x}([V])^{(V: U)}\right)_{[U] \in \mathcal{O}(G)}
$$

is a ring homomorphism. Here, $(V: U)$ represents $(G: U) /(G: V)$.

In this case, $\mathbb{W}_{G}(A)$ is called the Witt-Burnside ring of $G$ over $A$.

\section{Example 2.2.}

(a) If $G=\hat{C}$, the profinite completion of the multiplicative infinite cyclic group $C$, then the conjugacy classes of open subgroups are parametrized naturally by their index in $\hat{C}$. Thus, $\mathbb{W}_{G}$ coincides with $\mathbb{W}$, the functor of the ring of Witt vectors due to Witt and Lang $([5,8])$.

(b) If $G=\hat{C}_{p}$, the pro- $p$-completion of the infinite cyclic group, then $\mathbb{W}_{G}$ coincides with $\mathbb{W}_{p}$, the functor of the ring of $p$-typical Witt vectors due to Witt $([8,17])$.

(c) For a positive integer $n$ we denote by $\mathbf{n}$ the set of divisors of $n$. If $G$ is the finite cyclic group of order $n$, then $\mathbb{W}_{G}$ coincides with the functor of the ring of n-nested Witt vectors $([2])$.

Let us introduce a functor which is naturally equivalent to $\mathbb{W}_{G}$ on a category of special $\lambda$-rings. References for special $\lambda$-rings are $[1,9,13,15,16]$. Define a $\mathcal{O}(G) \times \mathcal{O}(G)$ matrix $\tilde{\zeta}_{G}$ by

$$
\tilde{\zeta}_{G}([V],[W])= \begin{cases}\varphi_{V}(G / W) \Psi^{(W: V)} & \text { if }[W] \preceq[V], \\ 0 & \text { otherwise. }\end{cases}
$$

Here, the notation $\Psi^{(W: V)}$ represents the $(W: V)$-th Adams operation.

Definition 2.3. ([15, 16]) Given a profinite group $G, N r_{G}$ is a unique covariant functor from the category of special $\lambda$-rings to the category of commutative rings with identity characterized as follows.

(1) As a set

$$
N r_{G}(A)=A^{\mathcal{O}(G)} .
$$

(2) For every special $\lambda$-ring homomorphism $f: A \rightarrow B$ and every $\alpha \in N r_{G}(A)$ one has

$$
N r_{G}(f)(\alpha)=f \circ \alpha
$$

(3) The map,

$$
\tilde{\varphi}: N r_{G}(A) \rightarrow \operatorname{Gh}(G, A), \quad \mathbf{x} \mapsto \tilde{\zeta}_{G} \mathbf{x},
$$

is a ring homomorphism. Here, we understand $\mathbf{x}$ as a $1 \times \mathcal{O}(G)$ column vector.

While the addition in $N r_{G}(A)$ is defined componentwise, the multiplication in $N r_{G}(A)$ is somewhat complicated. Given $\mathbf{x}, \mathbf{y} \in N r_{G}(A)$ and $[U] \in \mathcal{O}(G)$, the $[U]$-th component of $\mathbf{x} \cdot \mathbf{y}$ is given by

$$
\sum_{[V],[W] \in \mathcal{O}(G)} \sum_{\substack{V g W \subseteq G \\[Z(g, V, W)]=[U]}} \Psi^{(V: Z(g, V, W))}(\mathbf{x}([V])) \Psi^{(W: Z(g, V, W))}(\mathbf{y}([W])),
$$


where $Z(g, V, W)$ denotes $V \cap g W g^{-1}$.

Example 2.4. If $G$ is abelian, Eq. (2.1) is reduced to the following simple form

$$
\sum_{V, W \in \mathcal{O}(G)} \sum_{V \cap W=U}(G: V+W) \Psi^{(V: U))}(\mathbf{x}(V)) \Psi^{(W: U)}(\mathbf{y}(W)) .
$$

In particular, if $G=\hat{C}$, then

$$
\mathbf{x} \cdot \mathbf{y}(n)=\sum_{[i, j]=n}(i, j) \Psi^{\left(\frac{n}{d}\right)}(\mathbf{x}(d)) \Psi^{\frac{n}{d}}(\mathbf{y}(d)) .
$$

Here, $[i, j]$ represents the least common multiple and $(i, j)$ the greatest common divisor of $i$ and $j$.

Theorem 2.5. ([16]) Viewed as a functor from the category of special $\lambda$-rings to the category of commutative rings with identity, $\mathbb{W}_{G}$ is naturally isomorphic to $\mathrm{Nr}_{G}$.

Remark 2.6. A commutative ring $A$ with identity may have two different special $\lambda$-ring structures. Each of them will produce two different commutative rings, say $N r_{G, 1}(A)$ and $N r_{G, 2}(A)$. However, Theorem 2.5 says that they are isomorphic.

Define a $\mathcal{O}(G) \times \mathcal{O}(G)$ matrix $\zeta_{G}^{\dagger}$ by

$$
\zeta_{G}([V],[W])=\varphi_{V}(G / W)
$$

for all $[V],[W] \in \mathcal{O}(G)$. With this notation, we have

Definition 2.7. ([15]) Given a profinite group $G, \widehat{N r}_{G}$ is a unique covariant functor from the category of commutative rings with identity to itself characterized as follows.

(1) As a set

$$
\widehat{N r}_{G}(A)=A^{\mathcal{O}(G)} .
$$

(2) For every ring homomorphism $f: A \rightarrow B$ and every $\alpha \in \widehat{N r}_{G}(A)$ one has

$$
\widehat{N r}_{G}(f)(\alpha)=f \circ \alpha \text {. }
$$

(3) The map,

$$
\hat{\varphi}: \widehat{N r}_{G}(A) \rightarrow \operatorname{Gh}(G, A), \quad \mathbf{x} \mapsto \zeta_{G} \mathbf{x},
$$

is a ring homomorphism. Here, we understand $\mathbf{x}$ as a $1 \times \mathcal{O}(G)$ column vector.

Note that $\widehat{N r}_{G}(\mathbb{Z})$ is isomorphic to the Burnside-Grothendieck ring of almost finite $G$-spaces. The addition in $\widehat{N r}_{G}(A)$ is defined componentwise. On the other hand, the multiplication is defined as follows: Given $\mathbf{x}, \mathbf{y} \in \widehat{N r}_{G}(A)$ and $[U] \in$ $\mathcal{O}(G)$

$$
\mathbf{x} \cdot \mathbf{y}([U])=\sum_{[V],[W] \in \mathcal{O}(G)} \sum_{\substack{V, g W \subset G \\[Z(g, V, W)]=[U]}} \mathbf{x}([V]) \mathbf{y}([W])
$$

\footnotetext{
$\dagger$ In the literature this matrix is called the Burnside matrix of $G$.
} 
Example 2.8. If $G$ is abelian, Eq. (2.2) is reduced to

$$
\sum_{V, W \in \mathcal{O}(G)} \sum_{V \cap W=U}(G: V+W) \mathbf{x}(V) \mathbf{y}(W) .
$$

In particular, if $G=\hat{C}$, then

$$
\mathbf{x} \cdot \mathbf{y}(n)=\sum_{[i, j]=n}(i, j) \mathbf{x}(d) \mathbf{y}(d), \quad n \geq 1 .
$$

Indeed, $\widehat{N r}_{\hat{C}}$ was first introduced by Metropolis and Rota in order to describe Witt vectors $([12])$.

Recall that a special $\lambda$-ring in which $\Psi^{n}=i d$ for all $n \geq 1$ is called a binomial ring. The reason why binomial rings are important is that if $A$ is a binomial ring, then $N r_{G}(A)$ coincides with $\widehat{N r}_{G}(A)$. This follows from Eq. (2.1) and Eq. (2.2) immediately. Thus, viewed as a functor from the category of binomial rings to the category of commutative rings with identity, $N r_{G}$ and $\widehat{N r}_{G}$ will denote the same functor. Consequently we can conclude that

(1) $N r_{G} \cong \mathbb{W}_{G}$ as a functor on the category of special $\lambda$-rings, and

(2) $N r_{G}=\widehat{N r}_{G}$ as a functor on the category of binomial rings.

Remark 2.9. * Since we have necklace ring functors for both commutative rings with identity and special $\lambda$-rings, it would be quite natural to expect Witt-Burnside ring functors for both commutative rings with identity and special $\lambda$-rings, which has not, to the author's knowledge, been known yet. So, it seems quite challenging to construct a functor, say $\widehat{\mathbb{W}}_{G}$, satisfying

(1) $\widehat{\mathbb{W}}_{G} \cong \widehat{N r}_{G}$ on the category of special $\lambda$-rings, and

(2) $\widehat{\mathbb{W}}_{G}=\mathbb{W}_{G}$ on the category of binomial rings.

\section{ORBIT-SUM POLYNOMIALS ASSOCIATED WITH PROFINITE GROUPS}

Let $G$ be a profinite group and $X$ be an alphabet, that is, a set of commuting variables $\left\{x_{1}, x_{2}, \cdots, x_{m}\right\}$. Let us consider a discrete $G$-space (with respect to the discrete topology)

$$
\mathbb{Z} / q \mathbb{Z} \times X=\{(\bar{c}, x): \bar{c} \in \mathbb{Z} / q \mathbb{Z}, x \in X\}
$$

with trivial $G$-action. Denote by $\mathcal{C}(G, \mathbb{Z} / q \mathbb{Z} \times X)$ the set of all continuous maps from $G$ to $(\mathbb{Z} / q \mathbb{Z} \times X)$. It is well known that this set becomes a $G$-space with regard to the compact-open topology via the following standard $G$-action

$$
(g \cdot f)(a)=f\left(g^{-1} \cdot a\right) \quad \text { for all } a, g \in G
$$

(refer to [6]). For every open subgroup $V$ of $G$, the set of $V$-invariant elements in $\mathcal{C}(G, \mathbb{Z} / q \mathbb{Z} \times X)$ coincides with

$$
\operatorname{Hom}_{V}(G, \mathbb{Z} / q \mathbb{Z} \times X),
$$

the set of continuous $V$-maps from $G$ to $(\mathbb{Z} / q \mathbb{Z} \times X)$. If $f \in \operatorname{Hom}_{V}(G, \mathbb{Z} / q \mathbb{Z} \times X)$, then it is contained in an orbit isomorphic to $G / W$ for some $W$ to which $V$ is subconjugate. On the other hand, $(\mathbb{Z} / q \mathbb{Z} \times X)$ becomes a $\mathbb{Z} / q \mathbb{Z}$-set via the action

\footnotetext{
* The author is indebted to the referee for Remark 2.9.
} 
on the first component. Then $\mathcal{C}(G, \mathbb{Z} / q \mathbb{Z} \times X)$ also becomes a $\mathbb{Z} / q \mathbb{Z}$-set via the action

$$
(\bar{c} \cdot f)(x)=\left(\bar{c} \cdot\left(\pi_{1} \circ f\right)(x),\left(\pi_{2} \circ f\right)(x)\right), \quad(\bar{c} \in \mathbb{Z} / q \mathbb{Z}, x \in G) .
$$

Here, $\pi_{i},(i=1,2)$, represents the projection to the $i$-th component. With regard to $\mathbb{Z} / q \mathbb{Z}$-action we denote by

$$
\mathcal{C}(G, \mathbb{Z} / q \mathbb{Z} \times X) / \sim
$$

the set of the equivalence classes of all continuous maps from $G$ to $(\mathbb{Z} / q \mathbb{Z} \times X)$. In the natural way it becomes a $G$-set. Decompose $\mathcal{C}(G, \mathbb{Z} / q \mathbb{Z} \times X) / \sim$ into disjoint $G$-orbits and then consider its disjoint union, say,

$$
\bigcup_{h} G h .
$$

Here, $h$ runs through a system of representatives of this decomposition. Let $G_{h}$ be the isotropy subgroup of $h$. Write

$$
G=\bigcup_{1 \leq i \leq\left(G: G_{h}\right)} G_{h} w_{i}
$$

where $\left\{w_{i}: 1 \leq i \leq\left(G: G_{h}\right)\right\}$ is a fixed set of right coset representatives. With the above notation, we define $[h]$ by the polynomial in $x_{1}, x_{2}, \cdots, x_{m}$ given by

$$
\prod_{i=1}^{\left(G: G_{h}\right)}\left(\pi_{2} \circ h\right)\left(w_{i}\right) .
$$

It is not difficult to show that it is well defined, that is, it does not depend on the choice of coset representatives.

Definition 3.1. Let $h \in \operatorname{Hom}_{V}(G, \mathbb{Z} / q \mathbb{Z} \times X) / \sim$ and $W$ be an open subgroup of $G$. We say that $h$ has a period $W$ if

(1) $G_{h} \subseteq W$,

(2) There exists an element $h_{W} \in \operatorname{Hom}_{W}(G, \mathbb{Z} / q \mathbb{Z} \times X) / \sim$ and $s_{j} \in \mathbb{Z} / q \mathbb{Z}, 1 \leq$ $j \leq\left(W: G_{h}\right)$, such that

$$
h\left(t_{j} w_{i}\right)=s_{j} \cdot h_{W}\left(w_{i}\right)
$$

for $1 \leq i \leq(G: W), 1 \leq j \leq\left(W: G_{h}\right)$. Here, $\left\{w_{i}: 1 \leq i \leq(G: W)\right\}$ is a set of right-coset representatives of $W$ in $G$ and $\left\{t_{j}: 1 \leq j \leq\left(W: G_{h}\right)\right\}$ a set of right-coset representatives of $G_{h}$ in $W$.

If $h$ has a period $G_{h}$, then it is called aperiodic.

Definition 3.2. Let $q$ be any positive integer and $G$ be a profinite group.

(a) Given an open subgroup $V$ of $G$, we define $M_{G}^{q}(X, V)$, called the orbit-sum polynomial of $V$, by the polynomial in $x_{1}, x_{2}, \cdots, x_{m}$ given by

$$
\sum_{h}[h] \text {. }
$$

Here, $h$ ranges over the set of aperiodic representatives in the decomposition (3.1) such that $G$ h is isomorphic to $G / V$. 
(b) Let $f \in \operatorname{Hom}_{V}(G, \mathbb{Z} / q \mathbb{Z} \times X) / \sim$. Then the weight of $f$ over $V$, denoted by $\mathrm{wt}_{V}(f)$, is defined by

$$
\prod_{i=1}^{(G: V)}\left(\pi_{2} \circ f\right)\left(v_{i}\right)
$$

Here, $v_{i}$ 's range over a set of right-coset representatives of $V$ in $G$.

Define $\Psi^{n}$, the $n$-th Adams operation, on $\mathbb{Q}\left[x_{i}: 1 \leq i \leq m\right]$ as follows:

$$
\begin{aligned}
& \Psi^{n}\left(x_{i}\right)=x_{i}^{n}, \quad 1 \leq i \leq m, n \geq 1 \\
& \Psi^{n}(c)=c, \quad c \in \mathbb{Q} .
\end{aligned}
$$

The following lemma illustrates an intrinsic relation between orbit-sum polynomials and weights of functions.

Lemma 3.3. Let $q$ be any positive integer and $V$ be an open subgroup of $G$. Then the following identity holds.

$$
\sum_{f \in \operatorname{Hom}_{V}(G, \mathbb{Z} / q \mathbb{Z} \times X) / \sim} \operatorname{wt}_{V}(f)=\sum_{[W] \preceq[V]} \varphi_{V}(G / W) q^{(W: V)-1} \Psi^{(W: V)}\left(M_{G}^{q}(X, W)\right) .
$$

Proof. Decompose $\mathcal{C}(G, \mathbb{Z} / q \mathbb{Z} \times X) / \sim$ into disjoint $G$-orbits and consider its union, say,

$$
\bigcup_{h}^{\cdot} G h .
$$

Observe that $V$-invariant maps exist only in the orbits such that $G h$ are isomorphic to $G / W$ for some $W$ to which $V$ is subconjugate. For each aperiodic function $h$ such that $G h$ is isomorphic to $G / W$, note that there exist $q^{(W: V)-1}$-number of maps with period $h$ in $\operatorname{Hom}_{V}(G, \mathbb{Z} / q \mathbb{Z} \times X) / \sim$. On the other hand, every element in $\operatorname{Hom}_{V}(G, \mathbb{Z} / q \mathbb{Z} \times X) / \sim$ arises in this way. Since the number of all $V$-invariant elements in the orbit $G h$ is given by $\varphi_{V}(G / W)$ by definition and

$$
\mathrm{wt}_{V}(f)=[f]^{(W: V)}
$$

for all $V$-invariant functions $f \in G h$, the desired result follows.

Example 3.4. Note that

$$
\sum_{f \in \operatorname{Hom}_{V}(G, \mathbb{Z} / q \mathbb{Z} \times X) / \sim} \operatorname{wt}_{V}(f)=q^{(G: V)-1}\left(x_{1}+\cdots+x_{m}\right)^{(G: V)} .
$$

Thus, if $G=\hat{C}$ and $V=\hat{C}^{n}$ (a unique open subgroup of $\hat{C}$ of index $n$ ), then Eq. (3.2) is reduced to

$$
q^{n-1}\left(x_{1}+\cdots+x_{m}\right)^{n}=\sum_{d \mid n} d q^{\frac{n}{d}-1} \Psi^{\frac{n}{d}}\left(M_{G}^{q}\left(X, \hat{C}^{d}\right)\right) .
$$

From now on, we let $q$ be an indeterminate. Let us define a $\mathcal{O}(G) \times \mathcal{O}(G)$ matrix $\tilde{\zeta}_{G}^{q}$ by

$$
\tilde{\zeta}_{G}^{q}([V],[W])= \begin{cases}\varphi_{V}(G / W) q^{(W: V)-1} \Psi^{(W: V)} & \text { if }[W] \preceq[V] \\ 0 & \text { otherwise. }\end{cases}
$$


We also define a $\mathcal{O}(G) \times \mathcal{O}(G)$ matrix $\zeta_{G}^{q}$ by

$$
\zeta_{G}^{q}([V],[W])= \begin{cases}\varphi_{V}(G / W) q^{(W: V)-1} & \text { if }[W] \preceq[V] \\ 0 & \text { otherwise. }\end{cases}
$$

From the fact that $\varphi_{V}(G / W)=0$ unless $[W] \preceq[V]$ it follows that $\tilde{\zeta}_{G}^{q}$ is a lowertriangular matrix with the diagonal elements $\left(N_{G}(V): V\right) \cdot \mathrm{Id}$, the index of $V$ in its normalizer $N_{G}(V)$ in $G$, for $[V] \in \mathcal{O}(G)$. Therefore, $\tilde{\zeta}_{G}^{q}$ is invertible over a ring $A$ with identity if and only if $\left(N_{G}(V): V\right) \cdot 1$ is a unit in $A$ for all $[V] \in \mathcal{O}(G)$. Assume that the base ring is $\mathbb{Q}[q]$. Let $\tilde{\mu}_{G}^{q}$ (resp. $\mu_{G}^{q}$ ) be the inverse of $\tilde{\zeta}_{G}^{q}$ (resp. $\left.\zeta_{G}^{q}\right)$. Let us investigate how to compute the inverse of $\tilde{\mu}_{G}^{q}$ and $\mu_{G}^{q}$. First, we let $\mathcal{O}(G, U)$ be the subset of $\mathcal{O}(G)$ consisting of the elements satisfying the condition $[V] \preceq[U]$. Set

$$
\tilde{\mu}_{G, U}^{q}:=\left(\tilde{\zeta}_{G, U}^{q}\right)^{-1} \quad\left(\operatorname{resp}, \mu_{G, U}^{q}:=\left(\zeta_{G, U}^{q}\right)^{-1}\right),
$$

where $\tilde{\zeta}_{G, U}^{q}$ (resp. $\zeta_{G, U}^{q}$ ) is the matrix obtained from $\tilde{\zeta}_{G}^{q}$ (resp. $\zeta_{G}^{q}$ ) by restricting the index to $\mathcal{O}(G, U)$. It is not difficult to show that for all $[V] \in \mathcal{O}(G)$

$$
\tilde{\mu}_{G}^{q}([V],[W])= \begin{cases}\tilde{\mu}_{G, V}^{q}([V],[W]) & \text { if }[W] \in \mathcal{O}(G, V), \\ 0 & \text { otherwise. }\end{cases}
$$

Similarly, one can show that

$$
\mu_{G}([V],[W])= \begin{cases}\mu_{G, V}([V],[W]) & \text { if }[W] \in \mathcal{O}(G, V), \\ 0 & \text { otherwise. }\end{cases}
$$

The next lemma shows the relation between $\tilde{\mu}_{G}^{q}$ and $\mu_{G}^{q}$.

Lemma 3.5. For every $[W],[V] \in \mathcal{O}(G)$ satisfying $[W] \preceq[V]$, we have

$$
\tilde{\mu}_{G}^{q}([V],[W])=\mu_{G}^{q}([V],[W]) \Psi^{(W: V)} .
$$

Proof. Consider the following linear system

$$
\mathbf{y}=\tilde{\zeta}_{G, V} \mathbf{x}
$$

for $\mathbf{x}, \mathbf{y} \in \prod_{i \in \mathcal{O}(G, V)} \mathbb{Q}\left[x_{i}: 1 \leq i \leq m\right]$. This linear system can be rewritten as

$$
\tilde{\mathbf{y}}=\zeta_{G, V} \mathbf{x}
$$

where

$$
\tilde{\mathbf{y}}([W])=\Psi^{(W: V)}(\mathbf{y}([W])) \quad \text { for all }[W] \in \mathcal{O}(G, V) .
$$

Thus, we have the following equation:

$$
\mathbf{x}=\tilde{\mu}_{G, V} \mathbf{y}=\mu_{G, V} \tilde{\mathbf{y}} .
$$

Computing the $[V]$-th component from each side, we obtain

$$
\sum_{[W] \in \mathcal{O}(G, V)} \tilde{\mu}_{G, V}^{q}([V],[W]) \mathbf{y}([W])=\sum_{[W] \in \mathcal{O}(G, V)} \mu_{G, V}^{q}([V],[W]) \Psi^{(W: V)}(\mathbf{y}([W])) .
$$

This implies

$$
\tilde{\mu}_{G, V}^{q}([V],[W])=\mu_{G, V}^{q}([V],[W]) \Psi^{(W: V)} .
$$

So we are done. 
Theorem 3.6. Let $X=\left\{x_{1}, \cdots, x_{m}\right\}$ be an alphabet and $q$ be an indeterminate. Then, for a profinite group $G$ and an open subgroup $V$ of $G$, we have

$$
M_{G}^{q}(X, V)=\sum_{[W] \preceq[V]} \mu_{G}^{q}([V],[W]) q^{(G: W)-1}\left(x_{1}^{(W: V)}+\cdots+x_{m}^{(W: V)}\right)^{(G: W)} .
$$

Proof. Recall that

$$
\sum_{f \in \operatorname{Hom}_{V}(G, \mathbb{Z} / q \mathbb{Z} \times X) / \sim} \operatorname{wt}_{V}(f)=q^{(G: V)-1}\left(x_{1}+\cdots+x_{m}\right)^{(G: V)}
$$

(see Eq. (3.3)). From Lemma 3.3 it follows that

$$
\tilde{\zeta}_{G}^{q}\left(\begin{array}{c}
\vdots \\
M_{G}^{q}(X, V) \\
\vdots
\end{array}\right)=\left(\begin{array}{c}
\vdots \\
q^{(G: V)-1}\left(x_{1}+\cdots+x_{m}\right)^{(G: V)} \\
\vdots
\end{array}\right) .
$$

Taking $\tilde{\mu}_{G}^{q}$ on both sides and then applying Lemma 3.5 one can deduce the desired result.

Specializing $x_{i}$ into 1 for all $1 \leq i \leq m$, Eq. (3.6) is reduced to

$$
M_{G}^{q}(m, V)=\sum_{[W] \preceq[V]} \mu_{G}^{q}([V],[W]) q^{(G: W)-1} m^{(G: W)}
$$

which represents the number of aperiodic representatives $h$ such that $G h$ is isomorphic to $G / V$, in the decomposition (3.1). Replacing $m$ by an indeterminate $x$, we can obtain a polynomial in $x$ and $q$, denoted by $M_{G}^{q}(x, V)$.

Lemma 3.7. Suppose that a polynomial $f(x) \in \mathbb{Q}[x]$ takes integer values for all positive integers. Then it takes integer values for all integers.

Proof. Reducing to a common denominator we can write $f(x)=\frac{g(x)}{c}$ for some $g(x) \in \mathbb{Z}[x]$ and $c \in \mathbb{N}$. Suppose the assertion is false, then there must exist the largest integer $m_{0}$ such that $c$ does not divide $g\left(m_{0}\right)$. Then $c$ divides $g\left(m_{0}+c\right)$ by the maximal condition of $m_{0}$. But, since $g(x+c)=g(x)+c \cdot h(x)$ for some $h(x) \in \mathbb{Z}[x], c \mid g\left(m_{0}+c\right)$ implies that $c \mid g\left(m_{0}\right)$. This is a contradiction.

Lemma 3.7 says that $M_{G}^{q}(x, V)$ is a numerical polynomial in $q$ and $x$, that is, it takes integer values for all positive integers $q$ and $x$.

Remark 3.8. The orbit-sum polynomial $M_{G}^{q}(X, V)$ is a symmetric polynomial in $x_{i}$ 's. Hence, it can be written as a polynomial with integral coefficients in the elementary symmetric functions $e_{i}\left(x_{1}, \cdots, x_{m}\right), 1 \leq i \leq m$. On the other hand, all the coefficients are contained in $\mathbb{Q}[q]$. So, we can conclude that all of them are numerical polynomials in $q$.

In case where $G$ is abelian, we often prefer to using the matrix $\tilde{\zeta}_{\mathbf{G}}^{\mathbf{q}}$ instead of $\tilde{\zeta}_{G}^{q}$, which is defined by

$$
\tilde{\zeta}_{\mathbf{G}}^{\mathbf{q}}([V],[W])= \begin{cases}q^{(W: V)-1} \Psi^{(W: V)} & \text { if } V \subseteq W, \\ 0 & \text { otherwise. }\end{cases}
$$

Let $\tilde{\mu}_{\mathbf{G}}^{\mathbf{q}}$ be the inverse of $\tilde{\zeta}_{\mathbf{G}}^{\mathbf{q}}$. Then it is easy to show that

$$
\tilde{\mu}_{G}^{q}(V, W)=\frac{1}{(G: V)} \tilde{\mu}_{\mathbf{G}}^{\mathbf{q}}(V, W) .
$$


Similarly, if we define $\zeta_{\mathbf{G}}^{\mathbf{q}}$ by

$$
\zeta_{\mathbf{G}}^{\mathbf{q}}([V],[W])= \begin{cases}q^{(W: V)-1} & \text { if } V \subseteq W \\ 0 & \text { otherwise }\end{cases}
$$

and let $\mu_{\mathbf{G}}^{\mathbf{q}}$ be the inverse of $\zeta_{\mathbf{G}}^{\mathbf{q}}$, then it holds

$$
\mu_{G}^{q}(V, W)=\frac{1}{(G: V)} \mu_{\mathbf{G}}^{\mathbf{q}}(V, W) .
$$

Hence, Eq. (3.6) is reduced to

$$
M_{G}^{q}(X, V)=\frac{1}{(G: V)} \sum_{V \subseteq W} \mu_{\mathbf{G}}^{\mathbf{q}}(V, W) q^{(G: W)-1}\left(x_{1}^{(W: V)}+\cdots+x_{m}^{(W: V)}\right)^{(G: W)} .
$$

In the following, we would like to investigate $M_{G}^{q}(x, n)$ in detail in case where $G=\hat{C}$, the profinite completion of infinite cyclic group. Let $\hat{C}^{n}$ be a unique open subgroup of $\hat{C}$ of index $n$. The polynomial $M_{\hat{C}}^{q}\left(x, \hat{C}^{n}\right),(n \in \mathbb{N})$, first appeared in [11] in the context of formal group laws $F_{q}(X, Y)=X+Y-q X Y,(q \in \mathbb{Z})$. It was shown that they have a nice combinatorial description in terms of so called aperiodic q-words. Let us recall their definition briefly. First, consider the free monoid $(\mathbb{Z} / q \mathbb{Z} \times X)^{*}$ generated by $\mathbb{Z} / q \mathbb{Z} \times X$. Then, we define a $\mathbb{Z} / q \mathbb{Z}$-action on $(\mathbb{Z} / q \mathbb{Z} \times X)^{*}$ by letting the generator of $(\mathbb{Z} / q \mathbb{Z})$ act as

$$
\left(\left(r_{1}, a_{1}\right), \cdots,\left(r_{n}, a_{n}\right)\right) \mapsto\left(\left(r_{1}+1, a_{1}\right), \cdots,\left(r_{n}+1, a_{n}\right)\right) .
$$

We call $q$-words the orbits of this action. Let $\bar{w}$ denote the $q$-word with representative $w$. We call a positive integer $k$ a period if there is an element $w_{0}$ in $(\mathbb{Z} / q \mathbb{Z} \times X)^{*}$ of length $k$ and $r_{i}$ in $\mathbb{Z} / q \mathbb{Z}$ for $1 \leq i \leq|w| / k$, such that

$$
w=\left(r_{1} \cdot w_{0}\right) \cdots\left(r_{|w| / k} \cdot w_{0}\right) .
$$

Then aperiodic words are defined as usual. That is, $w$ is aperiodic if it is a word whose period equals its length. With this notation, we define

$$
[w]=\prod_{1 \leq i \leq n} a_{i}
$$

for $w=\left(\left(r_{1}, a_{1}\right), \cdots,\left(r_{n}, a_{n}\right)\right)$. Then it can be easily verified that

$$
M_{\hat{C}}^{q}\left(X, \hat{C}^{n}\right)=\sum_{w}[w],
$$

where the sum is over the equivalence classes of aperiodic $q$-words $w$ out of $X$ such that the length of $w$ is $n$. Moreover, if we specialize $x_{i}$ into 1 for all $i$, then Eq. (3.8) implies that $M_{\hat{C}}^{q}\left(m, \hat{C}^{n}\right)$ represents the number of the equivalence classes of aperiodic $q$-words $w$ out of $X$ such that the length of $w$ is equal to $n$. Use $M^{q}(X, n)$ instead of $M_{\hat{C}}^{q}\left(X, \hat{C}^{n}\right)$. Eq. (3.7) says that

$$
M^{q}(X, n)=\frac{1}{n} \sum_{d \mid n} \mu^{\mathbf{q}}(n, d) q^{d-1} p_{\frac{n}{d}}(X)^{d},
$$


YOUNG-TAK OH

where $\mu^{\mathbf{q}}(n, d)$ represents the $\left(\hat{C}^{n}, \hat{C}^{d}\right)$-th entry of the matrix $\mu_{\hat{\mathbf{C}}}^{\mathbf{q}}$. More precisely, $\zeta_{\hat{\mathbf{C}}}^{\mathbf{q}}$ is defined to be

$$
\zeta_{\hat{\mathbf{C}}}^{\mathbf{q}}\left(\hat{C}^{d_{1}}, \hat{C}^{d_{2}}\right)= \begin{cases}q^{\frac{d_{1}}{d_{2}}-1} & \text { if } d_{2} \mid d_{1}, \\ 0 & \text { otherwise }\end{cases}
$$

and $\mu_{\hat{\mathrm{C}}}^{\mathrm{q}}$ represents its inverse. Specializing $x_{i}$ into 1 for all $i$ and then replacing $m$ by $x$, Eq. (3.9) is reduced to

$$
M^{q}(x, n):=M_{\hat{C}}^{q}\left(x, \hat{C}^{n}\right)=\frac{1}{n} \sum_{d \mid n} \mu^{\mathbf{q}}(n, d) q^{d-1} x^{d} .
$$

\section{4. $q$-DEFORMATION OF NECKLACE RINGS}

In this section, we introduce a $q$-deformation of $N r_{G}$ and $\widehat{N r}_{G}$. Let $q$ be an indeterminate. For a special $\lambda$-ring $A$ consider the map,

$$
\tilde{\varphi}^{q}: A^{\mathcal{O}(G)} \rightarrow \mathrm{Gh}(G, A), \quad \mathbf{x} \mapsto \tilde{\zeta}_{G}^{q} \mathbf{x} .
$$

For the definition of $\tilde{\zeta}_{G}^{q}$ see Eq. (3.4). Note that we are using the column notation. It is obvious that if $A$ is a $\mathbb{Q}$-algebra, then $\tilde{\zeta}_{G}^{q}$ is invertible and

$$
\left(\tilde{\varphi}^{q}\right)^{-1}\left(\tilde{\varphi}^{q}(\mathbf{x})+\tilde{\varphi}^{q}(\mathbf{y})\right)=\mathbf{x}+\mathbf{y},
$$

where the addition $\mathbf{x}+\mathbf{y}$ is defined componentwise.

Lemma 4.1. Let $A$ be a special $\lambda$-ring equipped with $\mathbb{Q}$-algebra structure and $q$ an indeterminate. For every $[U] \in \mathcal{O}(G)$, set

$$
\mathfrak{p}_{U}^{q}:=\left(\tilde{\varphi}^{q}\right)^{-1}\left(\tilde{\varphi}^{q}(\mathbf{x}) \cdot \tilde{\varphi}^{q}(\mathbf{y})\right)([U])
$$

Then $\mathfrak{p}_{U}^{q}$ is expressed as

$$
\sum_{[V],[W] \preceq[U]} P_{V, W}^{U}(q) \Psi^{(V: U)}(\mathbf{x}([V])) \Psi^{(W: U)}(\mathbf{y}([W]))
$$

for some polynomials $P_{V, W}^{U}(q) \in \mathbb{Q}[q]$.

Proof. For all $[Z] \in \mathcal{O}(G)$ we have

$$
\begin{aligned}
& \left(\tilde{\zeta}_{G}^{q} \mathbf{x} \cdot \tilde{\zeta}_{G}^{q} \mathbf{y}\right)([Z]) \\
& =\sum_{\substack{[V] \leq[Z] \\
[W] \leq[Z]}} \varphi_{Z}(G / V) \varphi_{Z}(G / W) q^{(V: Z)-1} q^{(W: Z)-1} \Psi^{(V: Z)}(\mathbf{x}([V])) \Psi^{(W: Z)}(\mathbf{y}([W])) .
\end{aligned}
$$

Thus, the $[U]$-th component of $\tilde{\mu}_{G}^{q}\left(\tilde{\zeta}_{G}^{q} \mathbf{x} \cdot \tilde{\zeta}_{G}^{q} \mathbf{y}\right)$ is given by

$$
\begin{aligned}
& \sum_{[Z] \leq[U]} \tilde{\mu}_{G}^{q}([U],[Z]) \varphi_{Z}(G / V) \varphi_{Z}(G / W) q^{(V: Z)-1} q^{(W: Z)-1} \\
& \times\left(\sum_{\substack{[V] \leq[Z] \\
[W] \subseteq[Z]}} \Psi^{(V: Z)}(\mathbf{x}([V])) \Psi^{(W: Z)}(\mathbf{y}([W]))\right)
\end{aligned}
$$


Exploting Lemma 3.5 and the property $\Psi^{m} \circ \Psi^{n}=\Psi^{m n},(m, n \in \mathbb{N})$, we can rewrite Eq. (4.1) as

$$
\begin{aligned}
& \sum_{\substack{[Z] \leq[U] \\
[V][Z] \\
[W] \leq[Z] \\
[W] \leq}} \mu_{G}^{q}([U],[Z]) \varphi_{Z}(G / V) \varphi_{Z}(G / W) q^{(V: Z)-1} q^{(W: Z)-1} \\
& \quad \times \Psi^{(V: U)}(\mathbf{x}([V])) \Psi^{(W: U)}(\mathbf{y}([W])) .
\end{aligned}
$$

For $[V],[W] \preceq[U]$, set

$$
P_{V, W}^{U}(q):=\sum_{[V],[W] \preceq[Z] \preceq[U]} \mu_{G}^{q}([U],[Z]) \varphi_{Z}(G / V) \varphi_{Z}(G / W) q^{(V: Z)-1} q^{(W: Z)-1} .
$$

Clearly

$$
\mathfrak{p}_{U}^{q}=\sum_{[V],[W] \preceq[U]} P_{V, W}^{U}(q) \Psi^{(V: U)}(\mathbf{x}([V])) \Psi^{(W: U)}(\mathbf{y}([W])) .
$$

Since all the entries of $\mu_{G}^{q}$ are in $\mathbb{Q}[q]$, we can conclude that

$$
P_{V, W}^{U}(q) \in \mathbb{Q}[q] .
$$

Lemma 4.2. For every $[U],[V],[W] \in \mathcal{O}(G)$ satisfying $[V],[W] \preceq[U], P_{V, W}^{U}(q)$ is a numerical polynomial in $q$.

For the explicit form of $P_{V, W}^{U}(q)$ refer to Example 7.4. The proof of Lemma 4.2 will appear in Section 7. Lemma 4.2 has an amusing consequence that it yields a functor from the category of special $\lambda$-rings to the category of commutative rings. In particular, when $q=1$, it coincides with the functor $N r_{G}$ mentioned in Section 2 .

Theorem 4.3. Let $q$ be an integer and $G$ a profinite group. Then there exists a unique covariant functor $N r_{G}^{q}$ from the category of special $\lambda$-rings to the category of commutative rings satisfying the following conditions:

(1) As a set

$$
N r_{G}^{q}(A)=A^{\mathcal{O}(G)}
$$

(2) For every special $\lambda$-ring homomorphism $f: A \rightarrow B$ and every $\mathbf{x} \in N r_{G}^{q}(A)$ one has

$$
N r_{G}^{q}(f)(\mathbf{x})=f \circ \mathbf{x}
$$

(3) The map,

$$
\tilde{\varphi}^{q}: N r_{G}^{q}(A) \rightarrow \operatorname{Gh}(G, A), \quad \mathbf{x} \mapsto \tilde{\zeta}_{G}^{q} \mathbf{x}
$$

is a ring homomorphism. Here, we understand $\mathbf{x}$ as a $1 \times \mathcal{O}(G)$ column vector.

Similarly, let us consider the map,

$$
\varphi^{q}: A^{\mathcal{O}(G)} \rightarrow \mathrm{Gh}(G, A), \quad \mathrm{x} \mapsto \zeta_{G}^{q} \mathbf{x} .
$$

For the definition of $\zeta_{G}^{q}$ see Eq. (3.5). If $A$ is a $\mathbb{Q}$-algebra, then $\tilde{\zeta}_{G}^{q}$ is invertible and

$$
\left(\varphi^{q}\right)^{-1}\left(\varphi^{q}(\mathbf{x})+\tilde{\varphi}^{q}(\mathbf{y})\right)=\mathbf{x}+\mathbf{y} .
$$

For every $[U] \in \mathcal{O}(G)$, set

$$
p_{U}^{q}:=\left(\varphi^{q}\right)^{-1}\left(\varphi^{q}(\mathbf{x}) \cdot \varphi^{q}(\mathbf{y})\right)([U]) .
$$


It is not difficult to show that $p_{U}^{q}$ is given by

$$
\sum_{[V],[W] \preceq[U]} P_{V, W}^{U}(q) \mathbf{x}([V]) \mathbf{y}([W])
$$

for $P_{V, W}^{U}(q)$ appearing in Lemma 4.1. Since $P_{V, W}^{U}(q)$ 's are numerical polynomials we can state the following theorem.

Theorem 4.4. Let $q$ be an integer and $G$ a profinite group. Then there exists a unique functor $\widehat{N r}_{G}^{q}$ from the category of commutative rings with identity to the category of commutative rings satisfying the following conditions:

(1) As a set

$$
\widehat{N r}_{G}^{q}(A)=A^{\mathcal{O}(G)} .
$$

(2) For every ring homomorphism $f: A \rightarrow B$ and every $\mathbf{x} \in \widehat{N r}_{G}^{q}(A)$ one has

$$
\widehat{N r}_{G}^{q}(f)(\mathbf{x})=f \circ \mathbf{x} \text {. }
$$

(3) The map,

$$
\hat{\varphi}^{q}: \widehat{N r}_{G}^{q} \rightarrow \mathrm{Gh}(G, A), \quad \mathbf{x} \mapsto \zeta_{G}^{q} \mathbf{x},
$$

is a ring homomorphism.

As in the classical case, $N r_{G}^{q}$ and $\widehat{N r}_{G}^{q}$ will be equivalent on the category of binomial rings.

Example 4.5. Let $G$ be an abelian profinite group. Then the homomorphism $\tilde{\varphi}^{q}: N r_{G}^{q}(A) \rightarrow \mathrm{Gh}(G, A)$ is given by

$$
\mathbf{x} \mapsto\left(\sum_{U \subseteq V}(G: V) q^{(V: U)-1} \Psi^{(V: U)}(\mathbf{x}(V))\right)_{U \in \mathcal{O}(G)},
$$

and $\varphi^{q}: N r_{G}^{q}(A) \rightarrow \operatorname{Gh}(G, A)$ is given by

$$
\mathbf{x} \mapsto\left(\sum_{U \subseteq V}(G: V) q^{(V: U)-1} \mathbf{x}(V)\right)_{U \in \mathcal{O}(G)} .
$$

Remark 4.6. Recall that, in case $q=1, \widehat{N r}_{G}(\mathbb{Z})$ is isomorphic to the complete Burnside ring $\hat{\Omega}(G)([6])$. Similarly, we can realize $\widehat{N r}_{G}^{q}(\mathbb{Z})$ in terms of twisted Burnside ring $\hat{\Omega}^{q}(G)$. The underlying set of $\hat{\Omega}^{q}(G)$ is same to $\hat{\Omega}(G)$. Let $X$ and $Y$ be almost finite $G$-sets. Then, from the observation

$$
\tilde{\varphi}_{U}^{q}(X)=\varphi_{U}((\mathbb{Z} / q \mathbb{Z} \times X) / \sim),
$$

it follows that

$$
\begin{aligned}
& {[X] \oplus[Y]=[(\mathbb{Z} / q \mathbb{Z} \times X) / \sim]+[(\mathbb{Z} / q \mathbb{Z} \times Y) / \sim]} \\
& {[X] \otimes[Y]=[(\mathbb{Z} / q \mathbb{Z} \times X) / \sim] \cdot[(\mathbb{Z} / q \mathbb{Z} \times Y) / \sim],}
\end{aligned}
$$

where $\oplus, \otimes($ resp.,$+ \cdot)$ are operations in $\widehat{N r}_{G}^{q}(\mathbb{Z})$ (resp. $\hat{\Omega}(G)$ ). Extending these operations to $\hat{\Omega}(G)$ we obtain a ring. Denote this ring by $\hat{\Omega}^{q}(G)$. By construction

$$
\hat{\Omega}^{q}(G) \cong \widehat{N r}_{G}^{q}(\mathbb{Z}) \text {. }
$$




\section{5. $q$-INDUCTIONS AND $q$-RESTRICTIONS ON $N r^{q}$}

Let $G$ be a profinite group and $U$ an open subgroup of $G$. In this section we introduce two natural transformations

$$
\begin{aligned}
& q-\operatorname{Ind}_{U}^{G}: N r_{U}^{q} \rightarrow N r_{G}^{q}, \\
& q-\operatorname{Res}_{U}^{G}: N r_{G}^{q} \rightarrow N r_{U}^{q},
\end{aligned}
$$

which may be viewed as a $q$-version of inductions and restrictions at $q=1$. For the case where $q=1$ refer to $[13,14]$. We start by reviewing the classical case. References are $[13,14,16]$.

5.1. $q$-induction. Let $A$ be a special $\lambda$-ring $A$. Then the classical induction,

$$
\operatorname{Ind}_{U}^{G}: N r_{U}(A) \rightarrow N r_{G}(A), \quad \mathbf{x} \mapsto \operatorname{Ind}_{U}^{G}(\mathbf{x}),
$$

is defined so that the $[W]$-th component of $\operatorname{Ind}_{U}^{G}(\mathbf{x})([W])$ equals

$$
\sum_{\substack{[V] \in \mathcal{O}(U) \\[V]=[W] \text { in } \mathcal{O}(G)}} \mathbf{x}([V]) .
$$

Denoting by $I_{U}^{G}$ the matrix representing $\operatorname{Ind}_{U}^{G}$, it is immediate that

$$
I_{U}^{G}=\left(a_{[W],[V]}\right)_{[W] \in \mathcal{O}(G),[V] \in \mathcal{O}(U)},
$$

where

$$
a_{[W],[V]}= \begin{cases}1 & \text { if }[V]=[W] \text { in } \mathcal{O}(G), \\ 0 & \text { otherwise. }\end{cases}
$$

Example 5.1. If $G$ is abelian, then

$$
a_{W, V}= \begin{cases}1 & \text { if } V=W \\ 0 & \text { otherwise }\end{cases}
$$

In particular, when $G=\hat{C}$, the operator

$$
V_{r}:=I_{\hat{C}^{r}}^{\hat{C}}
$$

is given by the matrix $\left(a_{i, j}\right)_{i, j \in \mathbb{N}}$ where

$$
a_{i, j}:= \begin{cases}1 & \text { if }(i, j) \text { is of the form }(n r, n) \\ 0 & \text { otherwise }\end{cases}
$$

We now define $q$-induction

$$
q-\operatorname{Ind}_{U}^{G}: N r_{U}^{q} \rightarrow N r_{G}^{q}
$$

in two steps. First We will define induction

$$
\nu_{U}^{G}: \operatorname{Gh}(U, \cdot) \rightarrow \mathrm{Gh}(G, \cdot)
$$

satisfying

$$
\tilde{\varphi} \circ \operatorname{Ind}_{U}^{G}=\nu_{U}^{G} \circ \tilde{\varphi} .
$$

Once $\nu_{U}^{G}$ is defined, we will show that every entry of the matrix representing

$$
\left(\tilde{\varphi}^{q}\right)^{-1} \circ \nu_{U}^{G} \circ \tilde{\varphi}^{q}
$$

takes its value in $\mathbb{Z}$. Finally, we will define $q-\operatorname{Ind}_{U}^{G}$ by the multiplication by this matrix. 
Step 1:

Assume that $A$ is a $\mathbb{Q}$-algebra. Note that the matrix representing

$$
\tilde{\varphi}^{-1} \circ \operatorname{Ind}_{U}^{G} \circ \tilde{\varphi}: N r_{U} \rightarrow N r_{G}
$$

is given by

$$
\tilde{\zeta}_{G} I_{U}^{G} \tilde{\mu}_{U} \quad\left(\stackrel{\text { def }}{=}\left(c_{[W],[V]}\right)_{[W] \in \mathcal{O}(G),[V] \in \mathcal{O}(U)}\right) .
$$

Let

$$
\left\{\varepsilon_{[V]}:[V] \in \mathcal{O}(U)\right\} \quad\left(\text { resp. }\left\{\varepsilon_{[W]}:[W] \in \mathcal{O}(G)\right\}\right)
$$

be the standard basis of $\operatorname{Gh}(U, A)$ (resp. $\operatorname{Gh}(G, A)$ ), that is,

$$
\varepsilon_{[Z]}\left(\left[Z^{\prime}\right]\right)=\delta_{[Z],\left[Z^{\prime}\right]} .
$$

Here, $\delta$ represents Kronecker's delta. Similarly, we denote by

$$
e_{[W]} \in N r_{G}(A) \quad\left(\text { resp. } e_{[V]} \in N r_{U}(A)\right)
$$

the inverse image of $\tilde{\varphi}$ for

$$
\varepsilon_{[W]} \quad\left(\operatorname{resp} . \varepsilon_{[V]}\right) .
$$

Lemma 5.2. ([13, page 21]) Let $A$ be a special $\lambda$-ring and also a $\mathbb{Q}$-algebra. Let $U$ be an open subgroup of $G$. Then,

$$
\operatorname{Res}_{W}^{U}\left(e_{[V]}\right)=0
$$

unless $[V] \preceq[W]$ in $\mathcal{O}(U)$.

Lemma 5.3. Let $W, U$ be open subgroups of $G$ and $V$ be an open subgroup of $U$. Consider a coset-space decomposition

$$
G=\bigcup_{i}^{\cdot} g_{i} U
$$

of $U$ in $G$, where $\left\{g_{i}: 1 \leq i \leq(G: U)\right\}$ is a set of coset representatives. If $W$ is conjugate to $V$ in $G$, then the number of $g_{i}$ 's satisfying the conditions,

(1) $g_{i}^{-1} W g_{i} \subseteq U$

(2) (ii) $\left[g_{i}^{-1} W g_{i}\right]=[V]$ in $\mathcal{O}(U)$,

is given by

$$
\left[N_{G}(V): N_{U}(V)\right] .
$$

Proof. To begin with, we recall the identity

$$
\left[N_{G}(V) / N_{U}(V)\right]=\left[N_{G}(V) U / U\right] .
$$

By assumption there exists an element $t \in G$ such that $W=t^{-1} V t$. For our goal it suffices to show that

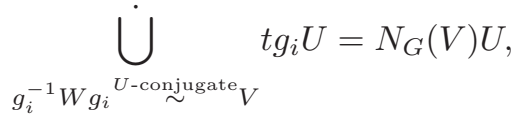

which can be easily verified.

In view of Lemma 5.2 and Lemma 5.3 we can establish the following significant result. 
Theorem 5.4. (cf. [3, Theorem 5.4.10]) Let $A$ be a special $\lambda$-ring and also a $\mathbb{Q}$ algebra. Suppose that $U$ is an open subgroup of $G$ and $V$ an open subgroup of $U$. Then

$$
\operatorname{Ind}_{U}^{G}\left(e_{[V]}\right)=\left[N_{G}(V): N_{U}(V)\right] e_{[V]},
$$

where the first $e_{[V]}$ is in $N r_{U}(A)$ and the second in $N r_{G}(A)$.

Proof. By the Mackey formula, for an open subgroup $W$ of $G$

$$
\begin{aligned}
& \operatorname{Res}_{W}^{G} \circ \operatorname{Ind}_{U}^{G}\left(e_{[V]}\right) \\
& =\sum_{W G \subseteq G} \operatorname{Ind}_{W \cap g U g^{-1}}^{W} \circ \operatorname{Res}_{W \cap g U g^{-1}}^{U}(g)\left(e_{[V]}\right) \\
& =\sum_{\substack{W g U \subseteq G \\
\left[W \cap g U g^{-1}\right] \succeq[V]}} \operatorname{Ind}_{W \cap g U g^{-1}}^{W} \circ \operatorname{Res}_{W \cap g U g^{-1}}^{U}(g)\left(e_{[V]}\right) \quad \text { (by Lemma 5.2). }
\end{aligned}
$$

Since the value of $\tilde{\varphi}_{W}$ on an element induced from a proper open subgroup of $W$ is zero,

$$
\begin{aligned}
& \tilde{\varphi}_{W}\left(\operatorname{Res}_{W}^{G} \circ \operatorname{Ind}_{U}^{G}\left(e_{[V]}\right)\right)=\tilde{\varphi}_{W}\left(\sum_{\substack{W g U \subseteq G \\
W \subseteq g U g \\
[g U g-1] \succeq[V]}} \operatorname{Res}_{g U g^{-1}}^{U}(g)\left(e_{[V]}\right)\right) \\
& = \begin{cases}\sum_{\substack{\left.W g U \subseteq G \\
W g \in G \\
g^{-1} W g \subseteq U \\
W g\right]=[V] \text { in } \mathcal{O}(U)}} 1 & \text { if } W \stackrel{\text { conjugate }}{\sim} V, \\
0 & \text { otherwise. }\end{cases} \\
& = \begin{cases}{\left[N_{G}(V): N_{U}(V)\right]} & \text { if } W \stackrel{\text { conjugate }}{\sim} V, \\
0 & \text { otherwise. }\end{cases}
\end{aligned}
$$

The last equality follows from Lemma 5.3. Hence, we have

$$
\operatorname{Ind}_{U}^{G}\left(e_{[V]}\right)=\left[N_{G}(V): N_{U}(V)\right] e_{[V]} .
$$

Theorem 5.4 implies that $\left(c_{[W],[V]}\right)$ is given by

$$
c_{[W],[V]}= \begin{cases}{\left[N_{G}(V): N_{U}(V)\right]} & \text { if }[V]=[W] \text { in } \mathcal{O}(G), \\ 0 & \text { otherwise. }\end{cases}
$$

Now, for an arbitrary special $\lambda$-ring $A$, define

$$
\nu_{U}^{G}: \operatorname{Gh}(U, A) \rightarrow \operatorname{Gh}(G, A)
$$

by

$$
\mathbf{x} \mapsto\left(c_{[W],[V]}\right) \mathbf{x}
$$

for all $\mathbf{x} \in \operatorname{Gh}(U, A)$. By definition it is straightforward that

$$
\tilde{\varphi} \circ \operatorname{Ind}_{U}^{G}=\nu_{U}^{G} \circ \tilde{\varphi} .
$$


Example 5.5. If $G$ is abelian, then

$$
c_{V, W}= \begin{cases}(G: U) & \text { if } W=V \\ 0 & \text { otherwise }\end{cases}
$$

In particular, when $G=\hat{C}$, the operator $V_{r}:=\nu_{\hat{C}^{r}}^{\hat{C}}$ is given by the matrix $\left(a_{i, j}\right)_{i, j \in \mathbb{N}}$ where

$$
c_{i, j}:= \begin{cases}r & \text { if }(i, j) \text { is of the form }(n r, n) \\ 0 & \text { otherwise }\end{cases}
$$

Step 2:

Now, let us define $q$-induction

$$
q-\operatorname{Ind}_{U}^{G}: N r_{U}^{q}(A) \rightarrow N r_{G}^{q}(A) .
$$

In case where $A$ is a $\mathbb{Q}$-algebra, it can be defined by

$$
\left(\tilde{\varphi}^{q}\right)^{-1} \circ \nu_{U}^{G} \circ \tilde{\varphi}^{q} .
$$

It is quite interesting to see that $q$-induction thus defined coincides with the classical one for every open subgroup $U$ of $G$. We need the following lemma.

\section{Lemma 5.6.}

(a) For open subgroups $W, U$ of $G$ we have

$$
\varphi_{W}(G / U)=\left[N_{G}(U): U\right] n(W, U)=\sum_{\substack{\left[W_{i}\right] \in \mathcal{O}_{(U)} \\\left[W_{i}\right]=[W] \text { in } \mathcal{O}(G)}}\left[N_{G}\left(W_{i}\right): N_{U}\left(W_{i}\right)\right],
$$

where $n(W, U)$ is the number of $G$-conjugates of $U$ containing $W$.

(b) For open subgroups $W, U$ of $G$ and an open subgroup $V$ of $U$, we have

$$
\varphi_{W}(G / V)=\sum_{\substack{\left[W_{i}\right] \in \mathcal{O}(U) \\\left[W_{i}\right]=[W] \text { in } \mathcal{O}(G)}}\left[N_{G}\left(W_{i}\right): N_{U}\left(W_{i}\right)\right] \varphi_{W_{i}}(U / V) .
$$

Proof. (a) In fact the first equality is well-known in case $G$ is finite (for example, see [4]), and it is easy to check that it is also true in case $G$ is an arbitrary profinite group. In detail, let $\left\{g_{i}: 1 \leq i \leq(G: U)\right\}$ be a complete set of left coset representatives of $U$ in $G$. Then $\varphi_{W}(G / U)$ is the number of $g_{i}$ 's satisfying

$$
W \subseteq g_{i} U g_{i}^{-1} .
$$

Note that $g_{i} \in g_{j} N_{G}(U)$ if and only if $g_{i} U g_{i}^{-1}=g_{j} U g_{j}^{-1}$. Hence, $\left[N_{G}(U): U\right]$ number of $g_{i}$ 's yields one $G$-conjugate. From this observation the first equality follows.

The second equality follows from (5.3). By definition of the induction map and $\tilde{\varphi}_{W}$ we have

$$
\tilde{\varphi} \circ \operatorname{Ind}_{U}^{G}\left(\varepsilon_{[U]}\right)=\varphi_{W}(G / U) .
$$

On the other hand, (5.3) yields that

$$
\nu_{U}^{G}\left(\tilde{\varphi}\left(\varepsilon_{[U]}\right)\right)([W])=\sum_{\substack{\left[W_{i}\right] \in \mathcal{O}_{(U)} \\\left[W_{i}\right]=[W] \text { in } \mathcal{O}_{(G)}}}\left[N_{G}\left(W_{i}\right): N_{U}\left(W_{i}\right)\right] .
$$

So we are done.

(b) can be proved in the exactly same way as in (a). 
Theorem 5.7. Let $A$ be a special $\lambda$-ring equipped with $\mathbb{Q}$-algebra structure. Then,

$$
q-\operatorname{Ind}_{U}^{G}=\operatorname{Ind}_{U}^{G}
$$

for every open subgroup $U$ of $G$ we have

Proof. It suffices to show

$$
\tilde{\varphi}^{q} \circ \operatorname{Ind}_{U}^{G}\left(\varepsilon_{[V]}\right)=\nu_{U}^{G} \circ \tilde{\varphi}^{q}\left(\varepsilon_{[V]}\right)
$$

for every $[V] \in \mathcal{O}(U)$. Note that for $[W] \in \mathcal{O}(G)$

$$
\begin{aligned}
& \tilde{\varphi}^{q} \circ \operatorname{Ind}_{U}^{G}\left(\varepsilon_{[V]}\right)([W]) \\
& =\varphi_{W}(G / V) q^{(V: W)-1} \\
& =\sum_{\substack{\left[W_{i}\right] \in \mathcal{O}(U) \\
\left[W_{i}\right]=[W] \text { in } \mathcal{O}(G)}}\left[N_{G}\left(W_{i}\right): N_{U}\left(W_{i}\right)\right] \varphi_{W_{i}}(U / V) q^{(V: W)-1} \text { (by Lemma 5.6) } \\
& =\nu_{U}^{G} \circ \tilde{\varphi}^{q}\left(\varepsilon_{[V]}\right)([W]) .
\end{aligned}
$$

So we are done.

Theorem 5.7 enables us to generalize $q$-induction for arbitrary special $\lambda$-rings in the obvious way. More precisely,

$$
q-\operatorname{Ind}_{U}^{G}: N r_{U}^{q}(A) \rightarrow N r_{G}^{q}(A), \quad \mathbf{x} \mapsto I_{U}^{G} \mathbf{x},
$$

where $I_{U}^{G}$ is the matrix representing $\operatorname{Ind}_{U}^{G}$. By definition it is obvious that

$$
\tilde{\varphi}^{q} \circ q-\operatorname{Ind}_{U}^{G}=\nu_{U}^{G} \circ \tilde{\varphi}^{q} .
$$

Similarly, if we define

$$
q-\operatorname{Ind}_{U}^{G}: \widehat{N r}_{U}^{q} \rightarrow \widehat{N r}_{G}^{q}
$$

by the multiplication by $I_{U}^{G}$, then

$$
\hat{\varphi}^{q} \circ q-\operatorname{Ind}_{U}^{G}=\nu_{U}^{G} \circ \hat{\varphi}^{q} .
$$

5.2. $q$-restriction. In this section, we investigate how to define $q$-restriction. In contrast with $q$-induction, $q$-restriction depends on $q$. First, we recall the classical case, i.e., the case where $q=1$. References are $[13,14]$. Given a special $\lambda$-ring $A$, restriction,

is defined by the assignment

$$
\operatorname{Res}_{U}^{G}: N r_{G}(A) \rightarrow N r_{U}(A), \quad \mathbf{x} \mapsto \operatorname{Res}_{U}^{G}(\mathbf{x}),
$$

$$
\operatorname{Res}_{U}^{G}(\mathbf{x})([W])=\sum_{[V] \in \mathcal{O}(G)} \sum_{[Z(g, U, V)]=[W]} \Psi^{(V: Z(g, U, V))}(\mathbf{x}([V])) .
$$

Here, $g$ ranges over the set of representatives of $U$-orbits of $G / V$ and $Z(g, U, V)$ stands for $U \cap g V g^{-1}$. Also, restriction on ghost rings is defined as follows:

$$
\mathcal{F}_{U}^{G}: \operatorname{Gh}(G, A) \rightarrow \operatorname{Gh}(U, A), \quad \mathbf{x} \mapsto R_{U}^{G} \cdot \mathbf{x},
$$

where $R_{U}^{G}$ represents the $\mathcal{O}(U) \times \mathcal{O}(G)$ matrix given by

$$
\left(b_{[V],[W]}\right)_{[V] \in \mathcal{O}(G),[W] \in \mathcal{O}(U)}
$$

with

$$
b_{[V],[W]}= \begin{cases}1 & \text { if }[W]=[V] \text { in } \mathcal{O}(G), \\ 0 & \text { otherwise }\end{cases}
$$


Lemma 5.8. ([13]) For any special $\lambda$-ring $A$,

$$
\tilde{\varphi}^{q} \circ \operatorname{Res}_{U}^{G}=\mathcal{F}_{U}^{G} \circ \tilde{\varphi}^{q} .
$$

Example 5.9. In case where $G$ is abelian, $R_{U}^{G}$ is given by the matrix $\left(b_{V, W}\right)_{V, W}$ with

$$
b_{V, W}= \begin{cases}1 & \text { if } V=W \\ 0 & \text { otherwise. }\end{cases}
$$

In particular, if $G=\hat{C}$,

$$
\begin{gathered}
\mathcal{F}_{\hat{C}^{r}}^{\hat{C}}: A^{\mathbb{N}} \rightarrow A^{\mathbb{N}} \\
\mathbf{x} \rightarrow R_{r} \mathbf{x},
\end{gathered}
$$

where $R_{r}\left(:=R_{\hat{C}}^{\hat{C}^{r}}\right)$ is given by the matrix $\left(a_{i, j}\right)_{i, j \in \mathbb{N}}$ with

$$
a_{i, j}= \begin{cases}1 & \text { if }(i, j) \text { is of the form }(n, n r) \\ 0 & \text { otherwise }\end{cases}
$$

Let us first assume that $A$ is a $\mathbb{Q}$-algebra. Set

$$
q-\operatorname{Res}_{U}^{G}=\left(\tilde{\varphi}^{q}\right)^{-1} \circ \mathcal{F}_{U}^{G} \circ \tilde{\varphi}^{q} .
$$

Lemma 5.10. Let $A$ be a special $\lambda$-ring equipped with $\mathbb{Q}$-algebra structure. For any two open subgroups $U, V$ of $G$, the composite map

$$
q-\operatorname{Res}_{U}^{G} \circ \operatorname{Ind}_{V}^{G}: N r_{V}^{q}(A) \rightarrow N r_{U}^{q}(A)
$$

is given by

$$
q-\operatorname{Res}_{U}^{G} \circ \operatorname{Ind}_{V}^{G}=\sum_{U g V \subseteq G} \operatorname{Ind}_{U \cap g V g^{-1}}^{U} \circ q-\operatorname{Res}_{U \cap g V g^{-1}}^{V}(g) .
$$

Proof. The left-hand side of Eq. (5.4) equals

$$
\begin{aligned}
q-\operatorname{Res}_{U}^{G} \circ \operatorname{Ind}_{V}^{G} & =\left(\tilde{\varphi}^{q}\right)^{-1} \circ \mathcal{F}_{U}^{G} \circ \tilde{\varphi}^{q} \circ \operatorname{Ind}_{V}^{G} \\
& =\left(\tilde{\varphi}^{q}\right)^{-1} \circ \mathcal{F}_{U}^{G} \circ \nu_{U}^{G} \circ \tilde{\varphi}^{q},
\end{aligned}
$$

and the right-hand side of Eq. (5.4) equals

$$
\begin{aligned}
& \sum_{U g V \subseteq G} \operatorname{Ind}_{U \cap g V g^{-1}}^{U} \circ q-\operatorname{Res}_{U \cap g V g^{-1}}^{V}(g) \\
= & \sum_{U g V \subseteq G} \operatorname{Ind}_{U \cap g V g^{-1}}^{U} \circ\left(\tilde{\varphi}^{q}\right)^{-1} \circ \mathcal{F}_{U \cap g V g^{-1}}^{V}(g) \circ \tilde{\varphi}^{q} \\
= & \sum_{U g V \subseteq G}\left(\tilde{\varphi}^{q}\right)^{-1} \circ \nu_{U \cap g V g^{-1}}^{U} \circ \mathcal{F}_{U \cap g V g^{-1}}^{V}(g) \circ \tilde{\varphi}^{q} .
\end{aligned}
$$

Thus, for our purpose, it suffices to show that

$$
\mathcal{F}_{U}^{G} \circ \nu_{U}^{G}=\sum_{U g V \subseteq G} \nu_{U \cap g V g^{-1}}^{U} \circ \mathcal{F}_{U \cap g V g^{-1}}^{V}(g) .
$$

This identity is also equivalent to

$$
\operatorname{Res}_{U}^{G} \circ \operatorname{Ind}_{V}^{G}=\sum_{U g V \subseteq G} \operatorname{Ind}_{U \cap g V g^{-1}}^{U} \circ \operatorname{Res}_{U \cap g V g^{-1}}^{V}(g),
$$

which follows from [13]. 
Consider the matrix representing the map $q-\operatorname{Res}_{U}^{G}$

$$
\left.\tilde{\mu}_{U}^{q} R_{U}^{G} \tilde{\zeta}_{U}^{q} \quad \stackrel{\stackrel{\text { def }}{=}}{=}\left(d_{[V],[W]}(q)\right)_{[V] \in \mathcal{O}(U),[W] \in \mathcal{O}(G)}\right) .
$$

By direct computation we obtain that

$$
d_{[V],[W]}(q)= \begin{cases}Q_{V, W}(q) \Psi^{(W: V)} & \text { if }[W] \preceq[V] \text { in } \mathcal{O}(G), \\ 0 & \text { otherwise }\end{cases}
$$

where

$$
Q_{V, W}(q)=\sum_{\substack{[S] \in \mathcal{O}(U) \\[W][S] \text { in } \mathcal{O}_{(G)} \\[S] \leq[V] \text { in } \mathcal{O}(U)}} \mu_{U}^{q}([V],[S]) \varphi_{S}(G / W) q^{(W: S)-1} .
$$

Theorem 5.11. Let $A$ be a special $\lambda$-ring with $\mathbb{Q}$-algebra structure. Then, $Q_{V, W}(q)$ is a numerical polynomial in $q$ for every $[V] \in \mathcal{O}(U)$ and every $[W] \in \mathcal{O}(G)$.

The proof of Theorem 5.11 will appear in Section 7.2. As a direct consequence of Theorem 5.11 we will extend $q$-restriction to arbitrary special $\lambda$-ring in the obvious way. More precisely, given a special $\lambda$-ring $A$, we define

$$
q-\operatorname{Res}_{U}^{G}: N r_{G}^{q}(A) \rightarrow N r_{U}^{q}(A)
$$

by

$$
\mathbf{x} \mapsto\left(d_{[V],[W]}(q)\right)(\mathbf{x}) .
$$

By definition it is obvious that

$$
\tilde{\varphi}^{q} \circ q-\operatorname{Res}_{U}^{G}=\mathcal{F}_{U}^{G} \circ \tilde{\varphi}^{q} .
$$

Similarly, if we define

$$
q-\operatorname{Res}_{U}^{G}: \widehat{N r}_{G}^{q} \rightarrow \widehat{N r}_{U}^{q}
$$

by the multiplication by the matrix

$$
\left(Q_{V, W}(q)\right)_{[V] \in \mathcal{O}(U),[W] \in \mathcal{O}(G)},
$$

then

$$
\hat{\varphi}^{q} \circ q-\operatorname{Res}_{U}^{G}=\mathcal{F}_{U}^{G} \circ \hat{\varphi}^{q} .
$$

6. $q$-DEFORMATION OF WitT-BURNSIDE RINGS

In this section, we construct a $q$-deformation of the Witt-Burnside ring of a profinite group, where $q$ ranges over the set of integers. Let $G$ be a profinite group. Put

$$
A=\mathbb{Q}[\mathbf{x}([U]), \mathbf{y}([U]):[U] \in \mathcal{O}(G)],
$$

where $\mathbf{x}([U]), \mathbf{y}([U])$ are indeterminates. Consider the map

$$
\begin{aligned}
& \Phi^{q}: A^{\mathcal{O}(G)} \rightarrow \operatorname{Gh}(G, A), \\
& \mathbf{x} \mapsto\left(\sum_{[V] \preceq[U]} \varphi_{U}(G / V) q^{(V: U)-1} \mathbf{x}([V])^{(V: U)}\right)_{[U] \in \mathcal{O}(G)} .
\end{aligned}
$$

For every $[U] \in \mathcal{O}(G)$ we let

$$
\mathfrak{s}_{[U]}:=\left(\Phi^{q}\right)^{-1}\left(\Phi^{q}(\mathbf{x})+\Phi^{q}(\mathbf{y})\right)([U])
$$

and

With this notation we have

$$
\mathfrak{p}_{[U]}:=\left(\Phi^{q}\right)^{-1}\left(\Phi^{q}(\mathbf{x}) \cdot \Phi^{q}(\mathbf{y})\right)([U]) .
$$


Lemma 6.1. Let $U$ be an open subgroup of $G$. Under the above hypothesis, we have

$$
\mathfrak{s}_{[U]}, \mathfrak{p}_{[U]} \in \mathbb{Z}[\mathbf{x}([V]), \mathbf{y}([V]):[V] \preceq[U]] .
$$

Furthermore, given $\mathbf{x} \in A^{\mathcal{O}(G)}$, there exists a unique $\iota_{\mathbf{x}}$ such that

$$
\iota_{\mathbf{x}}([U]) \in \mathbb{Z}[\mathbf{x}([V]):[V] \preceq[U]]
$$

and

$$
0=s_{[U]}\left(\mathbf{x}([V]), \iota_{\mathbf{x}}([V]):[V] \preceq[U]\right) .
$$

Example 6.2. Let $G$ be an abelian profinite group and $U$ an open subgroup of $G$ with $(G: U)=p$, where $p$ is a prime. Clearly $\mathfrak{s}_{G}=\mathbf{x}(G)+\mathbf{y}(G)$ and $\mathfrak{p}_{G}=\mathbf{x}(G) \mathbf{y}(G)$. Thus,

$$
\begin{aligned}
& \mathfrak{s}_{[U]}=\mathbf{x}(U)+\mathbf{y}(U)-\frac{q^{p-1}}{p} \sum_{r=1}^{p-1}\left(\begin{array}{l}
p \\
r
\end{array}\right) \mathbf{x}(G)^{r} \mathbf{y}(G)^{p-r}, \\
& \mathfrak{p}_{[U]}=\frac{q^{p-1}\left(q^{p-1}-1\right)}{p} \mathbf{x}(G)^{p} \mathbf{y}(G)^{p}+q^{p-1}\left(\mathbf{x}(G)^{p} \mathbf{y}(U)+\mathbf{x}(G) \mathbf{y}(G)^{p}\right)+p \mathbf{x}(U) \mathbf{y}(U) .
\end{aligned}
$$

The proof of Lemma 6.1 will appear in Section 7. In view of Lemma 6.1 one can derive a functor from the category of commutative rings with identity to the category of commutative rings.

Theorem 6.3. Let $q$ be an integer and $G$ be a profinite group. Then there exists a unique covariant functor $\mathbb{W}_{G}^{q}$ from the category of commutative rings with identity into the category of commutative rings satisfying the following conditions:

(1) As a set

$$
\mathbb{W}_{G}^{q}(A)=A^{\mathcal{O}(G)} .
$$

(2) For every ring homomorphism $f: A \rightarrow B$ and every $\alpha \in \mathbb{W}_{G}^{q}(A)$ one has

$$
\mathbb{W}_{G}^{q}(f)(\alpha)=f \circ \alpha .
$$

(3) The map,

$$
\begin{aligned}
& \Phi^{q}: \mathbb{W}_{G}^{q}(A) \rightarrow \operatorname{Gh}(G, A), \\
& \alpha \mapsto\left(\sum_{[G] \preceq[V] \preceq[U]} \varphi_{U}(G / V) q^{(V: U)-1} \alpha([V])^{(V: U)}\right)_{[U] \in \mathcal{O}(G)},
\end{aligned}
$$

is a ring homomorphism.

From the third condition it follows that

$$
\Phi^{q}(\alpha)=\frac{1}{q} \Phi(q \alpha) \quad \text { if } q \neq 0 .
$$

Here, $q \alpha$ denotes the vector whose $[U]$-th component is $q \alpha([U])$. This identity remains effective even when $q=0$ since

$$
\Phi(q \alpha)([U])
$$

is divided by $q$ for every $[U] \in \mathcal{O}(G)$. 
Example 6.4. If $G$ is abelian, then $\Phi^{q}: \mathbb{W}_{G}^{q}(A) \rightarrow \operatorname{Gh}(G, A)$ is given by

$$
\mathbf{x} \mapsto\left(\sum_{U \subseteq V}(G: V) q^{(V: U)-1} \mathbf{x}(V)^{(V: U)}\right) .
$$

In particular, if $G=\hat{C}$, then $\Phi^{q}: \mathbb{W}_{\hat{C}}^{q}(A) \rightarrow A$ is given by

$$
\mathbf{x} \mapsto\left(\sum_{d \mid n} d q^{\frac{n}{d}-1} \mathbf{x}(d)^{\frac{n}{d}}\right) .
$$

Denote by $\mathrm{D}(G)$ the set $\left\{\left(N_{G}(U): U\right):[U] \in \mathcal{O}(G)\right\}$ and by $\mathbb{Z}_{G}$ the commutative ring

$$
\mathbb{Z}\left[\frac{1}{\left(N_{G}(U): U\right)}:[U] \in \mathcal{O}(G)\right] .
$$

It is not difficult to show the following fact.

Lemma 6.5. Let $G$ be a profinite group. Then we have the following characterizations.

(a) $\Phi_{G}^{q}(A)$ is injective $\Leftrightarrow \varphi_{G}^{q}(A)$ is injective $\Leftrightarrow A$ has no $\left\{\left(N_{G}(U): U\right):[U] \in\right.$ $\mathcal{O}(G)\}$-torsion.

(b) $\Phi_{G}^{q}(A)$ is surjective $\Leftrightarrow \varphi_{G}^{q}(A)$ is surjective $\Leftrightarrow \Phi_{G}^{q}(A)$ is bijective $\Leftrightarrow \varphi_{G}^{q}(A)$ is bijective $\Leftrightarrow A$ is a $\mathbb{Z}_{G \text {-algebra. }}$

In the classical case we have constructed an isomorphism

$$
\tau: \mathbb{W}_{G}(A) \rightarrow N r_{G}(A),
$$

called Teichmüller map, for every special $\lambda$-ring $A([13,16])$. In the following, we will introduce its $q$-deformation

$$
\tau^{q}: \mathbb{W}_{G}^{q}(A) \rightarrow N r_{G}^{q}(A) .
$$

To do this, let us first define $q$-exponential maps

$$
\tau_{q}^{G}: A \rightarrow N r_{G}^{q}(A)
$$

Given an element $r \in A$, write it as a sum of one-dimensional elements, say $r_{1}+$ $r_{2}+\cdots+r_{m}$. Then, from Eq. (3.6) we have

$$
M_{G}^{q}\left(\left\{r_{1}, r_{2}, \cdots, r_{m}\right\}, V\right)=\sum_{[W] \preceq[V]} \mu_{G}^{q}(V, W) q^{(G: W)-1} \Psi^{(G: W)}\left(r^{(W: V)}\right) .
$$

for every open subgroup $V$ of $G$. Set

$$
M_{G}^{q}(r, V)=M_{G}^{q}\left(\left\{r_{1}, r_{2}, \cdots, r_{m}\right\}, V\right) .
$$

From Eq. (6.3) it follows that it is well-defined, that is, it does not depend on the choice of decompositions of $r$ into one-dimensional elements.

Lemma 6.6. Let $A$ be a special $\lambda$-ring. Then, for every $r \in A, M_{G}^{q}(r, V) \in A$.

Proof. By definition $M_{G}^{q}(r, V)$ is a symmetric polynomial in $r_{i}$ 's. Hence, it is an integral polynomial in $\lambda^{k}(r), 1 \leq k \leq(G: V)$, which is well known in the context of symmetric functions. Since $\bar{\lambda}^{k}(r) \in A$ for all $r \in A$ and $k \geq 1$, we have the desired result. 
Set

$$
\tau_{q}^{G}(r)([V])=M_{G}^{q}(r, V)
$$

for all $[V] \in \mathcal{O}(G)$. Lemma 6.6 implies that $\tau_{q}^{G}(r)$ is an element of $N r_{G}^{q}(A)$ for all $r \in A$. Moreover,

$$
\tilde{\varphi}^{q} \circ \tau_{q}^{G}(r)([V])=q^{(G: V)-1} r^{(G: V)}
$$

since $\tilde{\varphi}^{q}$ represents the multiplication by $\tilde{\zeta}_{G}^{q}$ (see Theorem 4.3). Using $q$-Ind $\operatorname{In}_{U}^{G}$ and $\tau_{q}^{U}$ for all $[U] \in \mathcal{O}(G)$ simultaneously, we construct a $q$-analog of Teichmüller map as follows:

$$
\begin{aligned}
& \tau^{q}: \mathbb{W}_{G}^{q}(A) \rightarrow N r_{G}^{q}(A), \\
& \alpha \mapsto \sum_{[U] \in \mathcal{O}(G)} \operatorname{Ind}_{U}^{G} \circ \tau_{q}^{U}(\alpha([U])) .
\end{aligned}
$$

We claim that $\Phi^{q}=\tilde{\varphi}^{q} \circ \tau^{q}$. In proving this argument, the following proposition plays a crucial role.

Proposition 6.7. Let $A$ be a special $\lambda$-ring with $\mathbb{Q}$-algebra structure. Then, for every $[U] \in \mathcal{O}(G)$ and $r \in A$, the identity

$$
q-\operatorname{Res}_{U}^{G}\left(\tau_{q}^{G}(r)\right)=\tau_{q}^{U}\left(q^{(G: U)-1} r^{(G: U)}\right)
$$

holds.

Proof. From Eq. (6.4) and the definition of $\mathcal{F}_{U}^{G}$ (see Section 5.2), the $[W]$-th component of $\mathcal{F}_{U}^{G} \circ \tilde{\varphi}^{q} \circ \tau_{q}^{G}(r)$ is given by

$$
\begin{cases}q^{(G: V)-1} r^{(G: V)} & \text { if }[W]=[V] \text { in } \mathcal{O}(G), \\ 0 & \text { otherwise, }\end{cases}
$$

for all $[W] \in \mathcal{O}(G)$. Hence,

$$
\begin{aligned}
q-\operatorname{Res}_{U}^{G}\left(\tau_{q}^{U}(r)\right)([W]) \\
=\left(\tilde{\varphi}^{q}\right)^{-1} \circ \mathcal{F}_{U}^{G} \circ \tilde{\varphi}^{q}\left(\tau_{q}^{U}(r)\right)([W]) \\
=\sum_{[U] \preceq[Z] \preceq[W]} \mu_{U}^{q}([W],[Z]) q^{(G: Z)-1} \Psi^{(Z: W)}\left(r^{(G: Z)}\right) \\
=\sum_{[U] \preceq[Z] \preceq[W]} \mu_{U}^{q}([W],[Z]) q^{(U: Z)-1} \Psi^{(Z: W)}\left(\left(q^{(G: U)-1)} r^{(G: U)}\right)^{(U: Z)}\right) \\
=M_{U}^{q}\left(q^{(G: U)-1)} r^{(G: U)}, W\right) .
\end{aligned}
$$

This completes the proof.

Theorem 6.8. $\tau^{q}$ is bijective for every special $\lambda$-ring $A$.

Proof. For $\alpha, \beta \in \mathbb{W}_{G}^{q}(A)$ we assume that

$$
\sum_{[U] \in \mathcal{O}(G)} \operatorname{Ind}_{U}^{G} \circ \tau_{q}^{U}(\alpha([U]))=\sum_{[U] \in \mathcal{O}(G)} \operatorname{Ind}_{U}^{G} \circ \tau_{q}^{U}(\beta([U])) .
$$

Then, for every $[U] \in \mathcal{O}(G)$,

$$
\left(\sum_{\substack{[V] \in \mathcal{O}(G) \\[U] \in \mathcal{O}(V)}} M_{V}^{q}(\alpha([V]), U)\right)=\left(\sum_{\substack{[V] \in \mathcal{O}(G) \\[U] \in \mathcal{O}(V)}} M_{V}^{q}(\beta([V]), U)\right) .
$$


It is clear that if $V=G$, then $\alpha([G])=\beta([G])$. Now assume that $\alpha([V])=\beta([V])$ for all $V$ such that $(G: V)<(G: U)$. From (6.6) it follows that $\alpha([U])=\beta([U])$. Thus, $\alpha=\beta$, and which implies the injectiveness of $\tau^{q}$.

Next, we will show that $\tau^{q}$ is surjective. For any $\mathbf{a} \in N r_{G}^{q}(A)$ we would like to find an element $\mathbf{x} \in \mathbb{W}_{G}^{q}(A)$ satisfying

$$
\left(\sum_{\substack{[V] \in \mathcal{O}(G) \\[U] \in \mathcal{O}(V)}} M_{V}^{q}(\mathbf{x}([V]), U)\right)=\mathbf{a}([U])
$$

for every $[U] \in \mathcal{O}(G)$. If $U=G$, then $\mathbf{x}([G])=\mathbf{a}([G])$. Let us use mathematical induction on the index. Assume that we have found $\mathbf{x}([V])$ for all $[V] \in \mathcal{O}(G)$ such that $(G: V)<(G: U)$. Setting

$$
\mathbf{x}([U])=\mathbf{a}([U])-\left(\sum_{\substack{[V] \in \mathcal{O}(G),[V] \neq[U] \\[U] \in \mathcal{O}(V)}} M_{V}^{q}(\mathbf{x}([V]), U)\right)
$$

we have

$$
\left(\sum_{\substack{[V] \in \mathcal{O}(G) \\[U] \in \mathcal{O}(V)}} M_{V}^{q}(\mathbf{x}([V]), U)\right)=\mathbf{a}([U])
$$

since $M_{U}^{q}(\mathbf{x}([U]), U)=\mathbf{x}([U])$. In this way we can find $\mathbf{x}$ satisfying Eq. (6.7).

If $U$ and $V$ are open subgroups of $G$ and if $g \in G$ conjugates $U$ into $V$, we can induce $q$-restriction and $q$-induction $q-\operatorname{Res}_{U}^{V}(g): N r_{V}^{q}(A) \rightarrow N r_{U}^{q}(A)$ and $\operatorname{Ind}_{U}^{V}(g)$ : $N r_{U}^{q}(A) \rightarrow N r_{V}^{q}(A)$ from the embedding $U \hookrightarrow V$. With this notation, we can extend some classical facts on $q$-induction and $q$-restriction, which can be proved by exploiting the facts in [6, Section 2.11].

\section{Proposition 6.9.}

(a) (Frobenius reciprocity) For any two open subgroups $U, V$ of $G$ and $\mathbf{x} \in$ $N r_{G}^{q}(A)$ and $\mathbf{y} \in N r_{U}^{q}(A)$, one has

$$
\operatorname{Ind}_{U}^{G}(\mathbf{y}) \cdot \mathbf{x}=\operatorname{Ind}_{U}^{G}\left(\mathbf{y} \cdot q-\operatorname{Res}_{U}^{G}(\mathbf{x})\right) .
$$

(b) (Mackey subgroup theorem) For any two open subgroups $U, V$ of $G$, the composite map

$$
q-\operatorname{Res}_{U}^{G} \circ \operatorname{Ind}_{V}^{G}: N r_{V}^{q}(A) \rightarrow N r_{U}^{q}(A)
$$

is given by

$$
q-\operatorname{Res}_{U}^{G} \circ \operatorname{Ind}_{V}^{G}=\sum_{U g V \subseteq G} \operatorname{Ind}_{U \cap g V g^{-1}}^{U} \circ q-\operatorname{Res}_{U \cap g V g^{-1}}^{V}(g) .
$$

(c) For any two open subgroups $U, V$ of $G$,

$$
\tilde{\varphi}_{U}^{q} \circ \operatorname{Ind}_{V}^{G}=\sum_{g V \in(G / V)^{U}} \tilde{\varphi}_{U}^{q} \circ q-\operatorname{Res}_{U}^{V}(g) .
$$


Proof. The proofs can be done with small modification of those of [6, Section 2.11]. So we will prove only (c). Note that $\tilde{\varphi}_{U}^{q}=\tilde{\varphi}_{U}^{q} \circ q-\operatorname{Res}_{U}^{G}$ since

$$
\begin{aligned}
\tilde{\varphi}_{U}^{q} \circ q-\operatorname{Res}_{U}^{G}(\mathbf{x}) & =\mathcal{F}_{U}^{G} \circ \tilde{\varphi}^{q}(\mathbf{x})([U]) \\
& =\tilde{\varphi}^{q}(\mathbf{x})([U]) \quad\left(\text { by def of } \mathcal{F}_{U}^{G}\right) .
\end{aligned}
$$

Furthermore $\tilde{\varphi}_{U}^{q} \circ \operatorname{Ind}_{U \cap g V g^{-1}}^{U}=0$ unless $U=U \cap g V g^{-1}$, equivalently,

$$
g V \in(G / V)^{U} .
$$

Applying these two facts to Eq. (6.8) yields the desired result.

Theorem 6.10. The following diagram

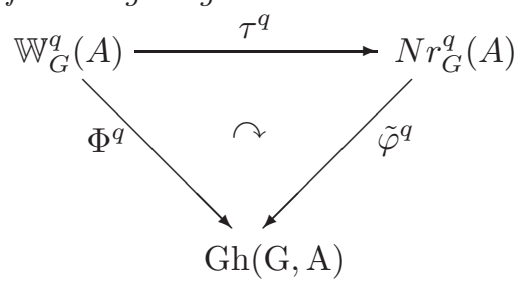

is commutative.

Proof. For $r \in A$,

$$
\begin{array}{rlrl}
\tilde{\varphi}_{U}^{q}\left(\operatorname{Ind}_{V}^{G} \circ \tau_{q}^{V}(r)\right) & =\sum_{g V \in(G / V)^{U}} \tilde{\varphi}_{U}^{q}\left(q-\operatorname{Res}_{U}^{V}(g)\left(\tau_{q}^{V}(r)\right) \quad \text { by }(6.9)\right. \\
& =\sum_{g V \in(G / V)^{U}} \tilde{\varphi}_{U}^{q}\left(\tau_{q}^{U}\left(q^{(V: U)-1} r^{(V: U)}\right)\right) & \text { by }(6.5) \\
& =\varphi_{U}(G / V) q^{(V: U)-1} r^{(V: U)} .
\end{array}
$$

Therefore, the additivity of $\tilde{\varphi}$ justifies our assertion.

Theorem 6.11. For every special $\lambda$-ring $A$, the $\operatorname{map} \tau^{q}: \mathbb{W}_{G}^{q}(A) \rightarrow N r_{G}^{q}(A)$ is a ring isomorphism.

Proof. We have already proved the bijectiveness of $\tilde{\varphi}$ in Theorem 6.8. In order to show that $\tilde{\varphi}$ is a ring homomorphism let $A=\mathbb{Q}[\mathbf{x}([U]), \mathbf{y}([U]):[U] \in \mathbb{O}(G)]$. Consider

$$
\tau^{q}(\mathbf{x}+\mathbf{y})=\tau^{q}(\mathbf{x})+\tau^{q}(\mathbf{y}) .
$$

We claim that the above identity is universal in the sense that, for every $[U] \in \mathcal{O}(G)$, the $[U]$-th component of both sides equals as a polynomial with integral coefficients. More precisely, Eq. (6.10) gives rise to the equality

$$
\sum_{[V] \in \mathcal{O}(G)} M_{G}^{q}((\mathbf{x}+\mathbf{y})([V]), U)=\sum_{[V] \in \mathcal{O}(G)}\left(M_{G}^{q}(\mathbf{x}([V]), U)+M_{G}^{q}(\mathbf{y}([V]), U)\right)
$$

for every $[U] \in \mathcal{O}(G)$. First, note that

$$
M_{G}^{q}(\mathbf{x}([V]), U), \quad[V],[U] \in \mathcal{O}(G),
$$

is a polynomial in $\lambda^{n}(\mathbf{x}([V])), n \geq 1$ with integral coefficients. Here, $\lambda^{n}$ represents the $n$-th $\lambda$-operation. Second, observe that

$$
(\mathbf{x}+\mathbf{y})([V]), \quad(\mathbf{x} \cdot \mathbf{y})([V]) \in \mathbb{Z}[\mathbf{x}([W]), \mathbf{y}([W]):[W] \preceq[V]] .
$$


(see Lemma 6.1) Putting these two facts together, we can say that

$$
M_{G}^{q}((\mathbf{x}+\mathbf{y})([V]), U), \quad M_{G}^{q}((\mathbf{x} \cdot \mathbf{y})([V]), U)
$$

are also polynomials in variables $\lambda^{n}(\mathbf{x}([V])), \lambda^{n}(\mathbf{y}([V])), n \geq 1$, with integral coefficients. Consequently, we can conclude that Eq. (6.11) is an identity between polynomials in $\lambda^{n}(\mathbf{x}([V])), \lambda^{n}(\mathbf{y}([V])), n \geq 1,[V] \preceq[U]$. Obviously this identity makes sense for arbitrary special $\lambda$-rings. In the same way as above we can show that

$$
\tau^{q}(\mathbf{x}+\mathbf{y})=\tau^{q}(\mathbf{x})+\tau^{q}(\mathbf{y})
$$

holds for arbitrary special $\lambda$-rings. So we are done.

Corollary 6.12. Let $A$ be a special $\lambda$-ring equipped with $\mathbb{Q}$-algebra structure. Then, for every $x, y \in A$, we have

$$
\tau_{q}^{G}(q x y)=q\left(\tau_{q}^{G}(x) \cdot \tau_{q}^{G}(y)\right) .
$$

Proof. Denote by $\varepsilon_{G}$ the vector determined by the condition $\varepsilon_{G}([W])=\delta_{[G],[W]}$. For every $x, y \in A$,

$$
\begin{aligned}
\tilde{\varphi}^{q}\left(q\left(\tau_{q}^{G}(x) \cdot \tau_{q}^{G}(y)\right)\right) & =q \Phi^{q}\left(x \delta_{G}\right) \Phi^{q}\left(y \delta_{G}\right) \\
& =\frac{1}{q} \Phi\left(q^{2} x y \delta_{G}\right) \\
& =\Phi^{q}\left(q x y \delta_{G}\right) \\
& =\tilde{\varphi}^{q}\left(\tau_{q}^{G}(q x y)\right) .
\end{aligned}
$$

The desired result follows from the injectiveness of $\varphi^{q}$.

The next corollary is almost straightforward.

Corollary 6.13. Let $A$ be a special $\lambda$-ring equipped with $\mathbb{Q}$-algebra structure. Then, as a ring,

$$
\mathbb{W}_{G}^{q}(A) \stackrel{\text { isomorphic }}{\cong} N r_{G}^{q}(A) \stackrel{\text { isomorphic }}{\cong} \widehat{N r}_{G}^{q}(A)
$$

Finally, we will define natural transformations $v_{U}^{q}, f_{U}^{q}$ on $\mathbb{W}_{G}^{q}$ by the transport of $q$-inductions and $q$-restrictions via the map $\tau^{q}$. First, in case $A=\mathbb{Z}$ we define

$$
\begin{aligned}
v_{U}^{q} & :=\left(\tau^{q}\right)^{-1} \circ \operatorname{Ind}_{U}^{G} \circ \tau^{q}, \\
f_{U}^{q} & :=\left(\tau^{q}\right)^{-1} \circ q-\operatorname{Res}_{U}^{G} \circ \tau^{q} .
\end{aligned}
$$

Theorem 6.14. Let $A=\mathbb{Z}$. Then for every $[U] \in \mathcal{O}(G)$, $v_{U}^{q}$ and $f_{U}^{q}$ are welldefined.

Proof. For $\alpha \in \mathbb{W}_{G}(\mathbb{Z})$ one has

$$
\begin{aligned}
& q-\operatorname{Res}_{U}^{G}\left(\tau^{q}(\alpha)\right) \\
& =\sum_{[V] \in \mathcal{O}(G)} q-\operatorname{Res}_{U}^{G} \cdot \operatorname{Ind}_{V}^{G}\left(\tau_{q}^{V}(\alpha([V]))\right) \\
& =\sum_{[V] \in \mathcal{O}(G)} \sum_{U g V \subseteq G} \operatorname{Ind}_{U}^{U} \cap g V g^{-1} \cdot q-\operatorname{Res}_{U}^{V} \cap g V G^{-1}(g)\left(\tau_{q}^{V}(\alpha([V]))\right) \\
& =\sum_{[V] \in \mathcal{O}(G)} \sum_{U g V \subseteq G} \operatorname{Ind}_{U}^{U} \cap g V g^{-1}\left(\tau_{q}^{U \cap g V g^{-1}}\left(\alpha([V])^{\left(V: U \cap g V g^{-1}\right)}\right)\right) .
\end{aligned}
$$


Hence, it follows from Lemma 7.7 that for any open subgroup $W$ of $U$

$$
\begin{aligned}
& \left(\tau^{q}\right)^{-1} \circ q-\operatorname{Res}_{U}^{G} \circ \tau^{q}(\alpha)([W]) \\
& =\xi_{\left(W ; W_{1}, \cdots, W_{k} ; 1, \cdots, 1\right)}^{G, q}\left(\alpha\left(\left[V_{1}\right]\right)^{\left(V_{1} ; W_{1}\right)}, \cdots, \alpha\left(\left[V_{k}\right]\right)^{\left(V_{k}, W_{k}\right)}\right),
\end{aligned}
$$

where $\xi_{\left(W ; W_{1}, \cdots, W_{k} ; 1, \cdots, 1\right)}^{G, q}$ is a polynomial with integral coefficients (For complete information refer to Lemma 7.7). Similarly, one can show $\left(\tau^{q}\right)^{-1} \circ \operatorname{Ind}_{U}^{G} \circ \tau^{q}(\alpha)([W])$ is a polynomial with integral coefficients in those $\alpha([V])$ 's, where $V$ ranges over the set of open subgroups of $U$ to which $W$ is sub-conjugate in $G$.

For an arbitrary commutative ring $A$, let us define restriction $f_{U}^{q}: \mathbb{W}_{G}^{q}(A) \rightarrow$ $\mathbb{W}_{U}^{q}(A)$ using the polynomials appearing in Eq. (6.12). This will give us a natural transformation $f_{U}^{q}: \mathbb{W}_{G}^{q} \rightarrow \mathbb{W}_{U}^{q}$ satisfying

$$
\tau^{q} \circ f_{U}^{q}=q-\operatorname{Res}_{U}^{G} \circ \tau^{q} .
$$

Similarly, one can define a natural transformation $v_{U}^{q}: \mathbb{W}_{U}^{q} \rightarrow \mathbb{W}_{G}^{q}$ such that

$$
\tau^{q} \circ v_{U}^{q}=\operatorname{Ind}_{U}^{G} \circ \tau^{q} .
$$

\section{LENART'S CONJECTURE AND PROOF OF LEMMAS AND THEOREMS}

7.1. Lenart's conjecture. In [11, page 731]) Lenart proposed a conjecture on $q$ restriction defined on $\operatorname{Nr}_{G}^{q}(A)$ in case where $G=\hat{C}$ (see Section 4). It can be stated as follows.

\section{Conjecture:}

Set

$$
f_{r}^{q}:=\mathcal{F}_{\hat{C}^{r}}^{\hat{C}}
$$

and

$$
M^{q}(x, n)=\sum_{d \mid n} \mu^{q}(n, d) q^{d-1} x^{d} .
$$

Then the $n$-th component of $f_{r}^{q}\left(M^{q}(x, n)_{n \geq 1}\right)$ is given by

$$
\sum_{d \mid n} Q_{r, n, d}(q) M^{q}\left(x^{r}, d\right) \quad(\text { in } \mathbb{Q}[x, q]),
$$

where $Q_{r, n, d}(q) \in \mathbb{Q}[q]$ are numerical polynomials.

Proof. Eq. (6.5) implies that

$$
f_{r}^{q} M^{q}(x)=M^{q}\left(q^{r-1} x^{r}\right) .
$$

If $r=1$, there is nothing to prove since $f_{1}^{q}$ is the identity map. For $r \geq 2$

$$
\begin{aligned}
M^{q}\left(q^{r-1} x^{r}\right) & =M^{q}\left(q \cdot q^{r-2} \cdot x^{r}\right) \\
& =q\left(M^{q}\left(q^{r-2}\right) \cdot M^{q}\left(x^{r}\right)\right),
\end{aligned}
$$


where the last equality follows from Corollary 6.12 or [11, Proposition 5.15]. Since

$$
\begin{aligned}
& q\left(M^{q}\left(q^{r-2}\right) \cdot M^{q}\left(x^{r}\right)\right)(n) \\
& =q \sum_{[i, j] \mid n}(i, j) P_{i, j}^{n}(q) M^{q}\left(q^{r-2}, i\right) M^{q}\left(x^{r}, j\right) \\
& =q \sum_{j \mid n}\left(\sum_{[i, j] \mid n}(i, j) P_{i, j}^{n}(q) M^{q}\left(q^{r-2}, i\right)\right) M^{q}\left(x^{r}, j\right),
\end{aligned}
$$

we obtain

$$
Q_{r, n, d}(q)=q\left(\sum_{[i, d] \mid n}(i, d) P_{i, d}^{n}(q) M^{q}\left(q^{r-2}, i\right)\right) .
$$

But, it was shown in [11] that $P_{i, d}^{n}(q)$ is a numerical polynomial. And $M^{q}\left(q^{r-2}, i\right)$ is also a numerical polynomial in view of Theorem 3.6. Thus, we can conclude that $Q_{r, n, d}(q)$ are numerical polynomials.

7.2. Proof of Theorem 5.11. Recall that the matrix representing $q-\operatorname{Res}_{U}^{G}$ is given by

$$
d_{[V],[W]}(q)= \begin{cases}Q_{V, W}(q) \Psi^{(W: V)} & \text { if }[W] \preceq[V] \text { in } \mathcal{O}(G) \\ 0 & \text { otherwise }\end{cases}
$$

where

$$
Q_{V, W}(q)=\sum_{\substack{[S] \in \mathcal{O}(U) \\[W]<[S] \text { in } \mathcal{O}(G) \\[S] \leq[V] \text { in } \mathcal{O}(U)}} \mu_{U}^{q}([V],[S]) \varphi_{S}(G / W) q^{(W: S)-1}
$$

Refer to Section 5. It is straightforward that $Q_{V, W}(q)$ is given by

$$
q-\operatorname{Res}_{U}^{G}\left(\varepsilon_{[W]}\right)([V])
$$

where $\varepsilon_{[W]}$ represents the vector determined by the condition

$$
\varepsilon_{[W]}\left(\left[W^{\prime}\right]\right)=\delta_{[W],\left[W^{\prime}\right]} .
$$

By Theorem 6.8 we can write $\varepsilon_{[W]}$ as

$$
\sum_{[U] \in \mathcal{O}(G)} \operatorname{Ind}_{U}^{G} \circ \tau_{q}^{V}(\alpha([V]))
$$

for some $\alpha \in A$. Now, from

$$
\mathbb{W}_{G}^{q}(\mathbb{Z}) \cong N r_{G}^{q}(\mathbb{Z})
$$

it follows that $\alpha([V]) \in \mathbb{Z}$ for all $[V] \in \mathcal{O}(G)$. Then, by Proposition 6.9 (b),

$$
\begin{aligned}
& q-\operatorname{Res}_{U}^{G} \circ\left(\sum_{[V] \in \mathcal{O}(G)} \operatorname{Ind}_{V}^{G} \circ \tau_{q}^{V}(\alpha([V]))\right) \\
& =\sum_{U g V \subseteq G} \sum_{[V] \in \mathcal{O}(G)} \operatorname{Ind}_{U \cap g V g^{-1}}^{U} \circ q-\operatorname{Res}_{U \cap g V g^{-1}}^{V}(g)\left(\tau_{q}^{V}(\alpha([V]))\right) \\
& =\sum_{U g V \subseteq G} \sum_{[V] \in \mathcal{O}(G)} \operatorname{Ind}_{U \cap g V g^{-1}}^{U}\left(\tau_{q}^{U \cap g V g^{-1}}\left(q^{\left(V: U \cap g V g^{-1}\right)-1} \alpha([V])^{\left(V: U \cap g V g^{-1}\right)}\right) .\right.
\end{aligned}
$$


The last equality follows from Eq. (6.5). Finally, our assertion follows from the fact that $M_{G}^{q}(x, V)$ is a numerical polynomial for every open subgroup $V$ of $G$.

\subsection{Proof of Lemma 4.2.}

Lemma 7.1. Let $n \in \mathbb{Z}_{>0}$ and $q \in \mathbb{Z}$. Write

$$
n=p_{1}^{a_{1}} \cdots p_{r}^{a_{r}} n^{\prime}, \quad q=p_{1}^{b_{1}} \cdots p_{r}^{b_{r}} q^{\prime}
$$

where $p_{i}$ 's are primes, $a_{i}, b_{i}>0$, and $\left(n^{\prime}, p_{i}\right)=\left(q^{\prime}, p_{i}\right)=\left(n^{\prime}, q^{\prime}\right)=1$ for all $i$. Then, $p_{i}^{a_{i}+b_{i}}$ divides $\frac{n}{d} q^{d}$ for all $1 \leq i \leq r$ and for all $d$ dividing $n$.

Proof. Write $d=p_{1}^{c_{1}} \cdots p_{r}^{c_{r}} d^{\prime}$ where $\left(p_{i}, d^{\prime}\right)=1$ for all $1 \leq i \leq r$. Then $p_{i}^{a_{i}-c_{i}} p_{i}^{b_{i}} p_{i}^{c_{i}}$ divides $\frac{n}{d} q^{d}$. But since $a_{i}-c_{i}+b_{i} p_{i}^{c_{i}} \geq a_{i}+b_{i}$ for all $1 \leq i \leq r$, we get the desired result.

Lemma 7.2. Let $q$ be a non-zero integer. For an open subgroup $V$ of $G$ let

$$
M_{G}^{q}(x, V):=\sum_{[W] \preceq[V]} \mu_{G}^{q}([V],[W]) q^{(G: W)-1} x^{(G: W)} .
$$

If $x=q^{m}$ for some positive integer $m$, then $\frac{1}{q} M_{G}^{q}(x, V)$ is a numerical polynomial in $q$.

Proof. In view of Lemma 3.3 and Theorem 3.6 we have

$$
q^{(G: V)-1} q^{m(G: V)}=\sum_{[W] \preceq[V]} \varphi_{V}(G / W) q^{(W: V)-1} M_{G}^{q}\left(q^{m}, W\right),
$$

equivalently

$$
\varphi_{V}(G / V) M_{G}^{q}\left(q^{m}, V\right)=q^{(G: V)-1} q^{m(G: V)}-\sum_{\substack{[W]<[V] \\[W] \neq[V]}} \varphi_{V}(G / W) q^{(W: V)} \frac{1}{q} M_{G}^{q}\left(q^{m}, W\right) .
$$

Note that $M_{G}^{q}\left(q^{m}, G\right)=q^{m}$. Hence, we can use an induction on the index $(G: V)$. Assume that our assertion holds for all $W$ 's satisfying $(G: W)>(G: V)$. Write

$$
\begin{aligned}
& \varphi_{V}(G / V)=p_{1}^{a_{1}} \cdots p_{r}^{a_{r}} \alpha^{\prime} \\
& q=p_{1}^{b_{1}} \cdots p_{r}^{b_{r}} q^{\prime},
\end{aligned}
$$

where $p_{i}$ 's are primes, $a_{i}, b_{i}>0$, and $\left(\alpha^{\prime}, q^{\prime}\right)=1$. Clearly $q^{\prime} \mid M_{G}^{q}\left(q^{m}, V\right)$ since $q$ divides the right hand side of Eq. (7.2). Recall that Corollary 5.6 implies that

$$
\varphi_{V}(G / W)=\sum_{\substack{\left[V_{i}\right] \in \mathcal{O}(W) \\\left[V_{i}\right]=[V] \text { in } \mathcal{O}(G)}}\left[N_{G}\left(V_{i}\right): N_{W}\left(V_{i}\right)\right] .
$$

Since $\left[N_{G}\left(V_{i}\right): N_{W}\left(V_{i}\right)\right] \mid \varphi_{V}(G / V)$ and $(W: V) \geq\left[N_{W}\left(V_{i}\right): V_{i}\right]$ we can obtain the desired result by applying Lemma 7.1.

Lemma 7.3. For every $[V],[W] \in \mathcal{O}(G)$ and for every $\alpha, \beta \in \mathbb{Z}$ one has the $q$ modified Mackey formula

$$
\begin{aligned}
& q\left(\operatorname{Ind}_{V}^{G}\left(\tau_{q}^{V}(\alpha)\right) \cdot \operatorname{Ind}_{W}^{G}\left(\tau_{q}^{W}(\beta)\right)\right) \\
& =\sum_{V g W \subseteq G} \operatorname{Ind}_{V \cap g W g^{-1}}^{G} \circ \tau_{q}^{V \cap g W g^{-1}} \\
& \quad\left(q^{-1}\left(q^{\left(V: V \cap g W g^{-1}\right)} \alpha^{\left(V: V \cap g W g^{-1}\right)}\right)\left(q^{\left(W: V \cap g W g^{-1}\right)} \beta^{\left(W: V \cap g W g^{-1}\right)}\right)\right) .
\end{aligned}
$$


Proof. If $q=0$, then it is trivial. So we assume that $q \neq 0$. We show that $\tilde{\varphi}_{U}^{q}$, applied to both sides of the above equation, yields the same number for all open subgroups $U$ in $G$. Observe

$$
\begin{aligned}
q & \left(\tilde{\varphi}_{U}^{q}\left(\operatorname{Ind}_{V}^{G}\left(\tau_{q}^{V}(\alpha)\right) \cdot \operatorname{Ind}_{W}^{G}\left(\tau_{q}^{W}(\beta)\right)\right)\right. \\
= & q\left(\tilde { \varphi } _ { U } ^ { q } \left(\operatorname{Ind}_{V}^{G}\left(\tau_{q}^{V}(\alpha)\right) \cdot \tilde{\varphi}_{U}^{q}\left(\operatorname{Ind}_{W}^{G}\left(\tau_{q}^{W}(\beta)\right)\right)\right.\right. \\
= & \left.q\left(\varphi_{U}(G / V) q^{(V: U)-1} \alpha^{(V: U)} \cdot \varphi_{U}(G / W) q^{(W: U)-1} \beta^{(W: U)}\right) \quad \text { (by Theorem } 6.10(\mathrm{~b})\right) \\
= & q\left(\varphi_{U}(G / V \times G / W)\left(q^{(V: U)-1} \alpha^{(V: U)}\right)\left(q^{(W: U)-1} \beta^{(W: U)}\right)\right) \\
= & \sum_{V g W \subseteq G} \varphi_{U}\left(G / V \cap g W g^{-1}\right) q^{\left(V \cap g W g^{-1}: U\right)-1} \\
& \times\left(q^{-1}\left(q^{\left(V: V \cap g W g^{-1}\right)} \alpha^{\left(V: V \cap g W g^{-1}\right)}\right)\left(q^{\left(W: V \cap g W g^{-1}\right)-1} \beta^{\left(W: V \cap g W g^{-1}\right)}\right)\right){ }^{\left(V \cap g W g^{-1}: U\right)} \\
= & \tilde{\varphi}_{U}^{q}\left(\sum_{V g W \subseteq G} \operatorname{Ind}_{V \cap g W g^{-1} \circ \tau_{q}^{\left(V \cap g W g^{-1}: U\right)}\left(q^{-1}\left(q^{\left(V: V \cap g W g^{-1}\right)} \alpha^{\left(V: V \cap g W g^{-1}\right)}\right)\right.}\right. \\
& \left.\left.\left(q^{\left(W: V \cap g W g^{-1}\right)} \beta^{\left(W: V \cap g W g^{-1}\right)}\right)\right)\right) .
\end{aligned}
$$

Main Proof. In case where $q=0$, our statement will be trivial since $\tilde{\varphi}^{0}$ is the identity map. Therefore, we assume that $q$ is not zero. In view of Lemma 4.1 we obtain that for every $[V] \in \mathcal{O}(G)$

$$
\varepsilon_{[V]} \cdot \varepsilon_{[W]}([U])= \begin{cases}P_{V, W}^{U}(q) & \text { if }[V],[W] \preceq[U] \\ 0 & \text { otherwise. }\end{cases}
$$

(see Eq. (7.1)). Note that

$$
\tau^{q}\left(\varepsilon_{[V]}\right)=\varepsilon_{[V]} \quad \text { and } \quad \tau^{q}\left(\varepsilon_{[W]}\right)=\varepsilon_{[W]},
$$

which can be shown by comparing the image of each side for $\tilde{\varphi}$. Eq. (7.3) implies that if $[V],[W] \preceq[U]$, then

$$
\begin{aligned}
& P_{V, W}^{U}(q) \\
& =\frac{1}{q} \sum_{V g W \subseteq G} \operatorname{Ind}_{V \cap g W g^{-1}}^{G} \tau_{q}^{V \cap g W g^{-1}}\left(q^{\left(V: V \cap g W g^{-1}\right)+\left(W: V \cap g W g^{-1}\right)-1}\right)([U]) .
\end{aligned}
$$

From Lemma 7.2 it follows that $P_{V, W}^{U}(q)$ is a numerical polynomial in $q$.

Example 7.4. If $G$ is abelian, then

$$
P_{V, W}^{U}(q)=\frac{1}{q}(G: V+W) M_{V \cap W}^{q}\left(q^{(V: V \cap W)+(W: V \cap W)-1}, U\right) .
$$

If $G=\hat{C}$ and $i, j \mid n$, then

$$
P_{\hat{C}^{i}, \hat{C}^{j}}^{\hat{C}^{n}}(q)=\frac{(i, j)}{q} M^{q}\left(q^{\frac{i+j}{(i, j)}-1}, \frac{n}{[i, j]}\right) .
$$


Remark 7.5. For all $[U],[V],[W]$ 's satisfying $[V],[W] \preceq[U], P_{V, W}^{U}(q)$ can also be computed recursively via the following formula:

$$
\sum_{[V],[W] \preceq[Z] \preceq[U]} \varphi_{U}(G / Z) q^{(Z: U)-1} P_{V, W}^{Z}(q)=\varphi_{U}(G / V) \varphi_{U}(G / W) q^{(V: U)+(W: U)-2} .
$$

Indeed, this identity follows from the fact that $\tilde{\varphi}^{q}$ is a ring homomorphism. In particular, if $U=Z(g, V, W)$, then

$$
P_{V, W}^{U}(q)=\frac{1}{\left(N_{G}(U): U\right)} \varphi_{U}(G / V) \varphi_{U}(G / W) q^{(V: U)+(W: U)-2} .
$$

\subsection{Proof of Theorem 6.1.}

Lemma 7.6. (cf. [6, Lemma (3.2.2)]) With the notation in [6, Lemma (3.2.2)], we obtain that for any $\alpha, \beta \in R$

$$
\tau_{q}^{G}(\alpha+\beta)=\sum_{G \cdot A \in G \backslash \mathfrak{U}(G)} \operatorname{Ind}_{U_{A}}^{G}\left(\tau_{q}^{U_{A}}\left(q^{-1}\left(q^{i_{A}} \alpha^{i_{A}} \cdot q^{i_{G-A}} \beta^{i_{G-A}}\right)\right)\right) .
$$

Proof. First we assume that $R$ is torsion-free. For every open subgroup $U$ of $G$, if we take $\tilde{\varphi}_{U}^{q}$ on the right side of Eq.(7.5), one has

$$
\begin{aligned}
& \tilde{\varphi}_{U}^{q}\left(\sum_{G \cdot A \in G \backslash \mathfrak{U}(G)} \operatorname{Ind}_{U_{A}}^{G}\left(\tau_{q}^{U_{A}}\left(q^{-1}\left(q^{i_{A}} \alpha^{i_{A}} \cdot q^{i_{G}-A} \beta^{i_{G-A}}\right)\right)\right)\right) \\
& =\sum_{\substack{G \cdot A \in G \mathfrak{U}(G) \\
U \lesssim U A}} \tilde{\varphi}_{U}\left(G / U_{A}\right) q^{\left(U_{A}: U\right)-1}\left(q^{-1}\left(q^{i_{A}} \alpha^{i_{A}} \cdot q^{i_{G-A}} \beta^{i_{G-A}}\right)\right){ }^{\left(U_{A}: U\right)} \text { (by Theorem } 6.10 \text { (b)) } \\
& =q^{\sharp(G / U)-1} \sum_{A \in \mathfrak{U}(G), U \lesssim U_{A}} \alpha^{\sharp(A / U)} \cdot \beta^{\sharp(G-A) / U} \\
& =q^{\sharp(G / U)-1}(\alpha+\beta)^{\sharp(G / U)} \\
& =\tilde{\varphi}_{U}^{q}\left(\tau_{q}^{G}(\alpha+\beta)\right) .
\end{aligned}
$$

Since $\tilde{\varphi}_{U}^{q}$ is injective, we have the desired result. In case $R$ is not torsion-free, $\tilde{\varphi}_{U}^{q}$ is no longer injective. However we note that the $U$-th component appearing in Eq. (7.5) is an integral polynomial in $\lambda^{k}\left(\alpha_{V}\right)$ 's and $\lambda^{l}\left(\beta_{W}\right)$ 's for $1 \leq k, l \leq[G: U]$ and $U \lesssim V, W \leqslant G$ for an arbitrary open subgroup $U$ of $G$. This implies that Eq. (7.5) holds regardless of torsion.

Lemma 7.7. (cf. $\left[6\right.$, Lemma (3.2.5)]) For some $k \in \mathbb{N}$ let $V_{1}, \cdots, V_{k} \leqslant G$ be a sequence of open subgroups of $G$. Then for every open subgroups $U \leqslant G$ and every sequence $\varepsilon_{1}, \cdots, \varepsilon_{k} \in\{ \pm 1\}$ there exists a unique polynomial $\xi_{U}^{q}=$ $\xi_{\left(U ; V_{1}, \cdots, V_{k} ; \varepsilon_{1}, \cdots, \varepsilon_{k}\right)}^{G, q}=\xi_{U}^{q}\left(x_{1}, \cdots, x_{k}\right) \in \mathbb{Z}\left[x_{1}, \cdots, x_{k}\right]$ such that for all $\alpha_{1}, \cdots, \alpha_{k} \in$ $\mathbb{Z}$ one has

$$
\left(\tau^{q}\right)^{-1}\left(\sum_{i=1}^{k} \varepsilon_{i} \cdot \operatorname{Ind}_{V_{i}}^{G}\left(\tau_{q}^{V_{i}}\left(\alpha_{i}\right)\right)(U)=\xi_{U}^{q}\left(\alpha_{1}, \cdots, \alpha_{k}\right) .\right.
$$

Proof. The proof can be done in the exactly same way of that of Lemma (3.2.5) [6]. Without loss of generality one may assume

$$
U \leqslant \bigcap_{i=1}^{k} V_{i}
$$


If $\varepsilon_{1}=\varepsilon_{2}=\cdots=\varepsilon_{k}=1$ and if $V_{i}$ is not conjugate to $V_{j}$ for $i \neq j$, then $\sum_{i=1}^{k} \varepsilon_{i} \operatorname{Ind}_{V_{i}}^{G}\left(\tau_{q}^{V_{i}}\left(\alpha_{i}\right)\right)=\tau^{q}(\alpha)$ for $\alpha \in \mathbb{W}_{G}^{q}(\mathbb{Z})$ with $[V]=\left[V_{j}\right]$ and $\alpha([V])=0$ if $V$ is not conjugate to either of the $V_{1}, \cdots, V_{k}$. So in this case we are done : $\xi_{U}^{q}\left(\alpha_{1}, \cdots, \alpha_{k}\right)$ equals $\alpha_{[U]}$. Hence, we may use triple induction, first with respect to

$$
m_{1}=m_{1}\left(U ; v_{1}, \cdots, V_{k} ; \varepsilon_{1}, \cdots, \varepsilon_{k}\right):=\max \left(\left(V_{i}: U\right) \mid \varepsilon_{i}=-1\right.
$$

or there exists some $j \neq i$ with $V_{j} \sim V_{i}$ ),

then with respect to

$$
m_{2}:=\sharp\left\{i \mid\left(V_{i}: U\right)=m_{1} \text { and } \varepsilon_{i}=-1\right\},
$$

and then with respect to

$$
m_{3}:=\sharp\left\{i \mid\left(V_{i}: U\right)=m_{1} \text { and there exists some } j \neq i \text { with } V_{j} \sim V_{i}\right\} \text {. }
$$

In case where $m_{1}=0$, the induction hypothesis holds in view of the above remark. In case where $m_{1}>0$, we have either $m_{2}>0$ or $m_{3}>0$. In case $m_{2}>0$, say $\left(V_{1}: U\right)=m_{1}$ and $\varepsilon_{1}=-1$, we may use Eq. (7.6) with $G=V_{1}, \alpha=-\alpha_{1}, \beta=+\alpha$ to conclude that

$$
0=\sum_{V_{1} \cdot A \in V_{1} \backslash \mathfrak{U}\left(V_{1}\right)} \operatorname{Ind}_{U_{A}}^{V_{1}}\left(\tau_{q}^{U_{A}}\left((-1)^{i_{A}} q^{\left(V_{1}: U_{A}\right)-1} \alpha_{1}^{\left(V_{1}: U_{A}\right)}\right)\right) .
$$

Therefore, considering the two special summands $A=\emptyset$ and $A=V_{1}$ and putting $\mathfrak{U}_{0}\left(V_{1}\right):=\left\{A \in \mathfrak{U}\left(V_{1}\right): A \neq \emptyset\right.$ and $\left.A \neq V_{1}\right\}$, one gets

$$
-\tau_{q}^{U_{A}}\left(\alpha_{1}\right)=\tau_{q}^{U_{A}}\left(-\alpha_{1}\right)+\sum_{V_{1} \cdot A \in V_{1} \backslash \mathfrak{U}_{0}\left(V_{1}\right)} \operatorname{Ind}_{U_{A}}^{V_{1}}\left(\tau_{q}^{U_{A}}\left((-1)^{i_{A}} \cdot q^{\left(V_{1}: U_{A}\right)-1} \alpha_{1}^{\left(V_{1}: U_{A}\right)}\right)\right) \text {. }
$$

Hence, if $A_{k+1}, A_{k+2}, \cdots, A_{k^{\prime}} \in \mathfrak{U}_{0}\left(V_{1}\right)$ denote representatives of the $V_{1}$-orbits $V_{1} \cdot A \subseteq \mathfrak{U}_{0}\left(V_{1}\right)$ with

$$
U \leqslant V_{k+1}:=U_{A_{k+1}}, V_{k+2}:=U_{A_{k+2}}, \cdots, V_{k^{\prime}}:=U_{A_{k^{\prime}}} \supsetneqq V_{1}
$$

and if we put $\varepsilon_{k+1}=\cdots=\varepsilon_{k^{\prime}}:=1$ and

$\alpha_{k+1}:=(-1)^{i_{A_{k+1}}} q^{\left(V_{1}: V_{k+1}\right)-1} \cdot \alpha_{1}^{\left(V_{1}: V_{k+1}\right)}, \cdots, \alpha_{k^{\prime}}:=(-1)^{i_{A_{k^{\prime}}}} q^{\left(V_{1}: V_{k^{\prime}}\right)-1} \cdot \alpha_{1}^{\left(V_{1}: V_{k^{\prime}}\right)}$,

then we have

$$
\begin{aligned}
& \xi_{U\left(U ; V_{1}, \cdots, V_{k} ;-1, \varepsilon_{2}, \cdots, \varepsilon_{k}\right)}^{q}\left(\alpha_{1}, \alpha_{2}, \cdots, \alpha_{k}\right) \\
& =\xi_{U\left(U ; V_{1}, \cdots, V_{k^{\prime}} ; 1, \varepsilon_{2}, \cdots, \varepsilon_{k^{\prime}}\right)}^{q}\left(-\alpha_{1}, \alpha_{2}, \cdots, \alpha_{k^{\prime}}\right),
\end{aligned}
$$

so the result follows by induction.

Similarly, if $m_{2}=0$, but $m_{3}>0$, say $V_{1}=V_{2}$, then we may use Eq. (7.6) once more with $G=V_{1}, \alpha=\alpha_{1}$, and $\beta=\alpha_{2}$ to conclude that

$$
\begin{aligned}
& \tau_{q}^{U_{A}}\left(\alpha_{1}+\alpha_{2}\right) \\
& =\sum_{V_{1} \cdot A \in V_{1} \backslash \mathfrak{U}\left(V_{1}\right)} \operatorname{Ind}_{U_{A}}^{V_{1}}\left(\tau_{q}^{U_{A}}\left(q^{\left(V_{1}: U_{A}\right)-1} \alpha^{i_{A}} \alpha_{2}^{i_{V_{1}-A}}\right)\right) \\
& =\tau_{q}^{U_{A}}\left(\alpha_{1}\right)+\tau_{q}^{U_{A}}\left(\alpha_{2}\right)+\sum_{V_{1} \cdot A \in V_{1} \backslash \mathfrak{U}_{0}\left(V_{1}\right)} \operatorname{Ind}_{U_{A}}^{V_{1}}\left(\tau_{q}^{U_{A}}\left(q^{\left(V_{1}: U_{A}\right)-1} \alpha_{1}^{i_{A}} \alpha_{1}^{i_{V_{1}}-A}\right)\right) .
\end{aligned}
$$

So with $V_{k+1}, \cdots, V_{k^{\prime}}$ as above, but $\varepsilon_{k+1}=\cdots=\varepsilon_{k^{\prime}}=-1$ and

$$
\alpha_{k+1}:=q^{\left(V_{1}: U_{A}\right)-1} \alpha_{1}^{i_{A_{k+1}}} \alpha_{2}^{i_{V_{1}-A_{k+1}}}, \cdots, \alpha_{k^{\prime}}:=q^{\left(V_{1}: U_{A}\right)-1} \alpha_{1}^{i_{A_{k^{\prime}}}} \alpha_{2}^{i_{V_{1}-A_{k^{\prime}}}},
$$


we get

$$
\begin{aligned}
& \xi_{U\left(U ; V_{1}, \cdots, V_{k} ; \varepsilon_{1}, \varepsilon_{2}, \cdots, \varepsilon_{k}\right)}^{q}\left(\alpha_{1}, \alpha_{2}, \cdots, \alpha_{k}\right) \\
& =\xi_{U\left(U ; V_{2}, \cdots, V_{k^{\prime}} ; \varepsilon_{2}, \cdots, \varepsilon_{k^{\prime}}\right)}^{q}\left(\alpha_{1}+\alpha_{2}, \alpha_{3}, \cdots, \alpha_{k^{\prime}}\right) .
\end{aligned}
$$

So again our result follows by induction since $A \in \mathfrak{U}_{0}\left(V_{1}\right)$ implies that $U_{A} \supsetneqq V_{1}$.

Main Proof. First, let us consider the equation

$$
\Phi^{q}(\mathbf{x})=\Phi^{q}(\alpha)+\Phi^{q}(\beta) .
$$

Applying the identity (6.1) yields that

$$
\begin{aligned}
\Phi^{q}\left(s^{q}\right) & =\frac{1}{q} \Phi(q \alpha)+\frac{1}{q} \Phi(q \beta) \quad(\text { in } \operatorname{Gh}(G, \mathbb{Z})) \\
& =\frac{1}{q} \Phi(q \alpha+q \beta) \quad\left(\text { in } \mathbb{W}_{G}(\mathbb{Z})\right) \\
& =\Phi^{q}\left(\frac{1}{q}(q \alpha+q \beta)\right) \quad\left(\text { in } \mathbb{W}_{G}(\mathbb{Z})\right)
\end{aligned}
$$

Thus, we get

$$
s_{U}^{q}=\frac{1}{q} s_{U}\left(q x_{V}, q y_{V}:[V] \preceq[U]\right) .
$$

Clearly $s_{U}^{q}$ is a polynomial in $\mathbb{Z}[\mathbf{x}([V]), \mathbf{y}([V]):[V] \preceq[U]]$ since $s_{U}$ has no constant term. Similarly,

$$
\begin{aligned}
-\Phi^{q}(\mathbf{a}) & =-\frac{1}{q} \Phi(q \mathbf{a}) \\
& =\frac{1}{q} \Phi(-q \mathbf{a}) \quad\left({ }^{\prime}-^{\prime} \text { means the inverse of }+ \text { in } \mathbb{W}(\mathbb{Z})\right) \\
& =\Phi^{q}\left(\frac{1}{q}(-q \mathbf{a})\right) .
\end{aligned}
$$

From this it follows that

$$
\iota_{[U]}^{q}=\frac{1}{q} \iota[U]((-q \mathbf{a})([V]):[V] \preceq[U]) .
$$

Clearly it has integer coefficients since $\iota_{[U]}$ has integer coefficients and no constant term.

To compute $p_{U}^{q}$ we use Eq. (7.3). First we choose a system $s_{1}, s_{2}, \cdots, s_{h}$ of representatives of the $G$-orbits in

$$
S:=\bigcup_{1 \leq i, j \leq k} G / V_{i} \times G / V_{j} .
$$

Next, we put $W_{r}:=G_{s_{r}}$ and

$$
p_{r}^{q}\left(x_{V_{1}}, y_{V_{1}}, \cdots, x_{V_{k}}, y_{V_{k}}\right):=q^{\left(V_{i}: W_{r}\right)-1} x_{i}^{\left(V_{i}: W_{r}\right)} q^{\left(V_{j}: W_{r}\right)-1} y_{i}^{\left(V_{j}: W_{r}\right)}
$$

in case $s_{r}=\left(g_{r} V_{i}, g_{r}^{\prime} V_{j}\right) \in G / V_{i} \times G / V_{j} \subseteq S$. Using these conventions and Eq. (7.3), we get the equation

$$
\begin{aligned}
& p_{U}^{q}\left(x_{V_{1}}, y_{V_{1}}, \cdots, x_{V_{k}}, y_{V_{k}}\right) \\
& =\xi_{\left(U ; W_{1}, \cdots, W_{h} ; 1 ; \cdots ; 1\right)}^{G, q}\left(p_{1}^{q}\left(x_{V_{1}} \cdots, y_{V_{k}}\right), \cdots, p_{h}^{q}\left(x_{V_{1}} \cdots, y_{V_{k}}\right)\right) .
\end{aligned}
$$

So, $p_{U}^{q}$ is also in $\mathbb{Z}[\mathbf{x}([V]), \mathbf{y}([V]):[V] \preceq[U]]$. 


\section{Classification $\mathbb{W}_{G}^{q}$ UP TO StRict NATURAL ISOMORPhism}

In this section, we assume that $G$ is an abelian profinite group. The aim of this section is to classify $\mathbb{W}_{G}^{q}$ up to strict natural isomorphism as $q$ ranges over the set of integers. To begin with, we introduce prerequisites. Given an integer $q$, we denote by $D(q)$ the set of divisors of $q$, and by $D^{\mathrm{pr}}(q)$ the set of prime divisors of $q$, respectively. Conventionally, $D(0)$ will denote the set of positive integers $\mathbb{N}$, and $D^{\mathrm{pr}}(0)$ the set of all primes in $\mathbb{N}$.

Definition 8.1. Let $q$ and $r$ be arbitrary integers.

(a) Given a commutative ring $A, \mathbb{W}_{G}^{q}(A)$ is said to be strictly-isomorphic to $\mathbb{W}_{G}^{r}(A)$ if there exists a ring isomorphism, say $\tau_{q}^{r}: \mathbb{W}_{G}^{q}(A) \rightarrow \mathbb{W}_{G}^{r}(A)$, satisfying $\Phi_{G}^{q}=\Phi_{G}^{r} \circ \tau_{q}^{r}$. In this case, $\tau_{q}^{r}$ is called a strict-isomorphism.

(b) $\mathbb{W}_{G}^{q}$ is said to be strictly-isomorphic to $\mathbb{W}_{G}^{r}$ if there exists a natural isomorphism, say $\tau_{q}^{r}: \mathbb{W}_{G}^{q} \rightarrow \mathbb{W}_{G}^{r}$, satisfying $\Phi_{G}^{q}=\Phi_{G}^{r} \circ \tau_{q}^{r}$. In this case, $\tau_{q}^{r}$ is called a strict natural isomorphism.

Denote by $\mathrm{D}^{\mathrm{pr}}(G)$ the set of prime divisors of each of $\{(G: U): U \in \mathcal{O}(G)\}$. And we let

$$
\begin{aligned}
& D^{\mathrm{pr}}(q) \cap D^{\mathrm{pr}}(G)=\left\{p_{1}, \cdots, p_{k} ; c_{1}, \cdots, c_{s}\right\}, \\
& D^{\mathrm{pr}}(r) \cap D^{\mathrm{pr}}(G)=\left\{p_{1}, \cdots, p_{k} ; d_{1}, \cdots, d_{t}\right\} .
\end{aligned}
$$

That is, $p_{i}$ 's are primes in $D^{\text {pr }}(q) \cap D^{\text {pr }}(r) \cap D^{\text {pr }}(G)$.

Theorem 8.2. Let $A$ be a commutative ring with identity. And, let $q, r$ be arbitrary integers and $G$ an abelian profinite group. Then, there exists a unique strictisomorphism between $\mathbb{W}_{G}^{q}(A)$ and $\mathbb{W}_{G}^{r}(A)$ if and only if $A$ is a $\mathbb{Z}\left[\frac{1}{c_{i}}, \frac{1}{d_{j}}: 1 \leq i \leq\right.$ $s, 1 \leq j \leq t]$-algebra

The above result will be proved in the following steps. First, let

$$
R=\mathbb{Q}\left[X_{U}: U \in \mathcal{O}(G)\right]
$$

and then consider the set of equations arising from

$$
\Phi_{G}^{q}(\mathbf{X})=\Phi_{G}^{r}(\mathbf{Y})
$$

More precisely, this set consists of the following identities

$$
\sum_{\substack{V \in \mathcal{O}(G) \\ U \subseteq V \subseteq G}}(G: V) q^{(V: U)-1} X_{V}^{(V: U)}=\sum_{\substack{V \in \mathcal{O}(G) \\ U \subseteq V \subseteq G}}(G: V) r^{(V: U)-1} Y_{V}^{(V: U)}
$$

for all $U \in \mathcal{O}(G)$. Here, $\mathbf{X}$ and $\mathbf{Y}$ represents the vectors $\left(X_{U}\right)_{U \in \mathcal{O}(G)}$ and $\left(Y_{U}\right)_{U \in \mathcal{O}(G)}$, respectively. Denote by $\mathbb{Z}_{G}$ the commutative $\operatorname{ring} \mathbb{Z}\left[\frac{1}{p}: p \in D^{\mathrm{pr}}(G)\right]$ (see Eq. $(6.2))$.

Lemma 8.3. Let $G$ be an arbitrary profinite group. Then, for every $U \in \mathcal{O}(G)$, it holds that

$$
Y_{U}-X_{U} \in\left(\mathbb{Z}_{G} \cap \mathbb{Z}\left[\frac{1}{q}, \frac{1}{r}\right]\right)\left[X_{S}: U \varsubsetneqq S \subseteq G, \text { and } S \in \mathcal{O}(G)\right] .
$$

Proof. Given $U \in \mathcal{O}(G)$ we would like to express $Y_{U}$ as a polynomial in $X_{V}$ 's inductively. In view of Eq. (8.1), $Y_{G}=X_{G}$. Now, we assume that

$$
Y_{V}-X_{V} \in \mathbb{Z}_{G}\left[X_{S}: V \varsubsetneqq S \subseteq G, \text { and } S \in \mathcal{O}(G)\right]
$$


for all $V \in \mathcal{O}(G)$ with $U \varsubsetneqq V \subseteq G$. Transform Eq. (8.1) into the form

$$
Y_{U}-X_{U}=\sum_{\substack{V \in \mathcal{O} G() \\ U \varsubsetneqq V \subseteq G}} \frac{\left(q^{(V: U)-1} X_{V}^{(V: U)}-r^{(V: U)-1} Y_{V}^{(V: U)}\right)}{(V: U)} .
$$

The induction hypothesis immediately implies that

$$
Y_{U}-X_{U} \in \mathbb{Z}_{G}\left[X_{S}: U \varsubsetneqq S \subseteq G \text {, and } S \in \mathcal{O}(G)\right] .
$$

Next, let us show that the coefficients are in $\mathbb{Z}\left[\frac{1}{q}, \frac{1}{r}\right]$. Let us first assume that $q$ and $r$ are nonzero. Multiply $q r /(q, r)$ to both sides of Eq. (8.1) to obtain the identity

$$
\Phi_{G}\left(\left(q X_{U}\right)_{U \in \mathcal{O}(G)}\right)=\Phi_{G}\left(\frac{q}{(q, r)} \cdot\left(r Y_{U}\right)_{U \in \mathcal{O}(G)}\right) .
$$

It follows from the injectiveness of $\Phi_{G}$ that

$$
\frac{r}{(q, r)} \cdot\left(q X_{U}\right)_{U \in \mathcal{O}(G)}=\frac{q}{(q, r)} \cdot\left(r Y_{U}\right)_{U \in \mathcal{O}(G)}
$$

It is not difficult to show that, for all $U \in[G]$, the $U$-th coordinates of $r /(q, r)$. $\left(q Y_{U}\right)_{U \in \mathcal{O}(G)}$ and $q /(q, r) \cdot\left(q X_{U}\right)_{U \in \mathcal{O}(G)}$ are of the form

$$
\frac{r q}{(q, r)} Y_{U}+\text { a polynomial contained in } \mathbb{Z}\left[q Y_{S}: U \varsubsetneqq S \subseteq G, \text { and } S \in \mathcal{O}(G)\right]
$$

and

$$
\frac{r q}{(q, r)} X_{U}+\text { a polynomial contained in } \mathbb{Z}\left[q X_{S}: U \varsubsetneqq S \subseteq G \text {, and } S \in \mathcal{O}(G)\right],
$$

respectively. Assume that

$$
Y_{V}-X_{V} \in \mathbb{Z}\left[\frac{1}{q}, \frac{1}{r}\right]\left[X_{S}: V \varsubsetneqq S \subseteq G, \text { and } S \in \mathcal{O}(G)\right]
$$

for all $V \in \mathcal{O}(G)$ with $U \varsubsetneqq V \subseteq G$. Applying the induction hypothesis above to Eq. (8.2) and Eq. (8.3) yields

$$
Y_{U}-X_{U} \in \mathbb{Z}\left[\frac{1}{q}, \frac{1}{r}\right]\left[X_{S}: U \varsubsetneqq S \subseteq G, \text { and } S \in \mathcal{O}(G)\right] .
$$

This completes the proof.

Lemma 8.4. Let $G$ be an abelian profinite group. Then, for every open subgroup $U$ of $G$, it holds that

$Y_{U}-X_{U} \in \mathbb{Z}\left[\frac{1}{c_{i}}, \frac{1}{d_{j}}: 1 \leq i \leq s, 1 \leq j \leq t\right]\left[X_{S}: U \varsubsetneqq S \subseteq G\right.$, and $\left.S \in \mathcal{O}(G)\right]$.

Proof. Let us first assume that $q$ and $r$ are nonzero. Note that $Y_{G}=X_{G}$. Now, we assume that

$$
Y_{V}-X_{V} \in\left(\mathbb{Z}_{G} \cap \mathbb{Z}\left[\frac{1}{q}, \frac{1}{r}\right]\right)\left[X_{S}: V \varsubsetneqq S \subseteq G, \text { and } S \in \mathcal{O}(G)\right]
$$

for all $V \in \mathcal{O}(G)$ with $U \varsubsetneqq V \subseteq G$. Since $G$ is abelian, Eq. (8.1) is transformed into

$$
Y_{U}-X_{U}=\sum_{\substack{V \in \mathcal{O}(G) \\ U \varsubsetneqq V \subseteq G}} \frac{q^{(V: U)-1} X_{V}^{(V: U)}-r^{(V: U)-1} Y_{V}^{(V: U)}}{(V: U)} .
$$


Note that if a prime $p$ divides $(V: U)$ and $q$ then it cannot be a divisor of the denominator of the irreducible fraction of $q^{(V: U)-1} /(V: U)$. Also, it cannot be a divisor of the denominator of the irreducible fraction of $r^{(V: U)-1} /(V: U)$ if it divides $r$. Now, our assertion follows from the induction hypothesis.

Next, we assume that $q$ is zero and $r$ is nonzero. In this case, Eq. (8.1) is reduced to

$$
Y_{U}-X_{U}=-\sum_{\substack{V \in \mathcal{O}(G) \\ U \varsubsetneqq V \subseteq G}} \frac{r^{(V: U)-1}}{(V: U)} Y_{V}^{(V: U)}
$$

Since a prime $p$ dividing $(V: U)$ and $r$ cannot be a divisor of the denominator of the irreducible fraction of $r^{(V: U)-1} /(V: U)$ we obtain the desired result by induction hypothesis. So we are done.

Proof of Theorem 8.2. The "if" part follows from Lemma 8.4. For the "only if" part, let us assume that there exists a unique strict-isomorphism, say $\tau_{q}^{r}: \mathbb{W}_{G}^{q}(A) \rightarrow \mathbb{W}_{G}^{r}(A)$. Hence, we have $\Phi_{G}^{q}=\Phi_{G}^{r} \circ \tau_{q}^{r}$. Assume that a prime $p$ divides $q$ and $(G: U)$ for some open subgroup $U$ of $G$, but not $r$. Consider a maximal filtration $G=V_{1} \supsetneqq V_{2} \supsetneqq \cdots \supsetneqq V_{k}=U$, where $V_{i}(1 \leq i \leq k)$ are open subgroups of $G$ such that there is no open subgroup between $V_{i}$ and $V_{i+1}$. Then $p$ must divide $\left(V_{i}: V_{i+1}\right)$ for some $1 \leq i \leq k$. Letting

$$
\left(a_{U}\right)_{U \in \mathcal{O}(G)}=\tau_{q}^{r}(0,0, \cdots, \overbrace{1}^{V_{i}-\text { th }}, 0,0, \cdots),
$$

we can deduce the identity

$$
q^{\left(V_{i}: V_{i+1}\right)-1}-r^{\left(V_{i}: V_{i+1}\right)-1}=\left(V_{i}: V_{i+1}\right) a_{V_{i+1}}
$$

by comparing the $V_{i+1}$-th component of both sides of Eq. (8.4). We claim that $p$ is a unit in $A$. To see this, transform Eq. (8.5) into

$$
-r^{\left(V_{i}: V_{i+1}\right)-1}=p\left(\frac{\left(V_{i}: V_{i+1}\right) a_{V_{i+1}}-q^{\left(V_{i}: V_{i+1}\right)-1}}{p}\right)
$$

Since $p$ and $r^{\left(V_{i}: V_{i+1}\right)-1}$ are coprime there are $x, y \in \mathbb{Z}$ such that

$$
p x-r^{\left(V_{i}: V_{i+1}\right)-1} y=1 .
$$

Therefore,

$$
-r^{\left(V_{i}: V_{i+1}\right)-1} y=1-p x=p\left(\frac{\left(V_{i}: V_{i+1}\right) a_{V_{i+1}}-q^{\left(V_{i}: V_{i+1}\right)-1}}{p}\right) y .
$$

Hence, $p\left(x+\left(\frac{\left(V_{i}: V_{i+1}\right) a_{\left[V_{i+1}\right]}-q^{\left(V_{i}: V_{i+1}\right)-1}}{p}\right) y\right)=1$. This justifies our claim. Similarly, a prime $p$ dividing $r$ and $(G: U)$, but not $q$, should be a unit. This implies that $A$ should be a $\mathbb{Z}\left[\frac{1}{c_{i}}, \frac{1}{d_{j}}: 1 \leq i \leq s, 1 \leq j \leq t\right]$-algebra.

From Theorem 8.2 it is immediate that $\mathbb{W}_{G}^{q}(\mathbb{Z})$ is classified up to strict-isomorphism by the set of prime divisors of $q$ contained in $D^{\text {pr }}(G)$. Thus, we proved

Corollary 8.5. Let $q$ vary over the set of integers and $G$ be an abelian profinite group. Then, $\mathbb{W}_{G}^{q}$ is classified up to strict natural isomorphism by the set of prime divisors of $q$ contained in $D^{\mathrm{pr}}(G)$. 
Acknowledgement The author would like to express his sincere gratitude to the referee for his/her correction of many errors in the previous version and valuable advices.

\section{REFERENCES}

[1] M. F. Atiyah, D. O. Tall, Group representations, $\lambda$-rings and the $J$-homomorphism, Topology 8 (1969), 253-297.

[2] R. Auer, A functorial property of nested Witt vectors, J. Alg. 252 (2002), 293-299.

[3] D. J. Benson, Representation and cohomology I, Cambridge Univ. Press, Cambridge, 1995.

[4] T. Tom Dieck, Transformation groups and representation theory, Lecture notes in Math. 766, Springer Verlag, (1979).

[5] J. Dieudonné, Schur functions and group representations, Astérisque 87-88 (1981), 7-19.

[6] A. Dress, C. Siebeneicher, The Burnside ring of profinite groups and the Witt vectors construction, Adv. Math. 70 (1988), 87-132.

[7] A. Dress, C. Siebeneicher, The Burnside ring of the infinite cyclic group and its relation to the necklace algebra, $\lambda$-ring and the Universal ring of the Witt vectors, Adv. Math. 78 (1989), 1-41.

[8] M. Hazewinkel, Formal groups and applications, Academic Press, New York, 1978.

[9] D. Knutson, $\lambda$-rings and the representation theory of the symmetric group, Lecture notes in Math. 308, Springer-Verlag, 1973.

[10] S. Lang, Algebra, -3rd ed., Addison-Wesley, Reading, MA, 1993.

[11] C. Lenart, Formal group-theoretic generalization of the necklace algebra, including a q-deformation, J. Alg. 199 (1998), 703-732.

[12] N. Metropolis, G.-C. Rota, Witt vectors and the algebra of necklaces, Adv. Math. 50 (1983), 95-125.

[13] Y. -T. Oh, $R$-analogue of the Burnside ring if profinite groups and free Lie algebras, Adv. Math. 190 (2005), 1-46.

[14] Y. -T. Oh, Corrigendum to R-analogue of the Burnside ring of profinite groups and free Lie algebras: [Adv. Math. 190 (2005) 1-46], Adv. Math. 192 (2005), 226-227.

[15] Y. -T. Oh, Generalized Burnside-Grothendieck ring functor and aperiodic ring functor associated with profinite groups, J. Alg. 291 (2005), no. 2, 607-648.

[16] Y. -T. Oh, Necklace rings and logarithmic functions, Adv. Math. 205 (2006), no. 2, 434-486.

[17] E. Witt, Zyklisch Körper und Algebren der Charakteristik $p$ vom Grade $p^{m}$, J. Reine Angew. Math. 176 (1937), 126-140.

Department of Mathematics, Sogang University, Seoul 121-742, Korea

E-mail address: ytoh@sogang.ac.kr 